\title{
Differentially Private Reinforcement Learning with Linear Function Approximation
}

\author{
Xingyu Zhou*
}

\begin{abstract}
Motivated by the wide adoption of reinforcement learning (RL) in real-world personalized services, where users' sensitive and private information needs to be protected, we study regret minimization in finite horizon Markov decision processes (MDPs) under the constraints of differential privacy (DP). Compared to existing private RL algorithms that work only on tabular finite-state, finite-actions MDPs, we take the first step towards privacy-preserving learning in MDPs with large state and action spaces. Specifically, we consider MDPs with linear function approximation (in particular linear mixture MDPs) under the notion of joint differential privacy (JDP), where the RL agent is responsible for protecting users' sensitive data. We design two private RL algorithms that are based on value iteration and policy optimization, respectively, and show that they enjoy sub-linear regret performance while guaranteeing privacy protection. Moreover, the regret bounds are independent of the number of states, and scale at most logarithmically with the number of actions, making the algorithms suitable for privacy protection in nowadays large scale personalized services. Our results are achieved via a general procedure for learning in linear mixture MDPs under changing regularizers, which not only generalizes previous results for non-private learning, but also serves as a building block for general private reinforcement learning.
\end{abstract}

\section{Introduction}

Reinforcement learning (RL) is a control-theoretic protocol, which adaptively learns to make sequential decisions in an unknown environment through trial and error. RL has shown to have significant success for delivering a wide variety of personalized services, including online news and advertisement recommendation (Li et al., 2010), medical treatment design (Zhao et al., 2009), natural language processing (Sharma and Kaushik, 2017), and social robot (Gordon et al., 2016). In these applications, an RL agent improves its personalization algorithm by interacting with users to maximize the reward. In particular, in each round, the RL agent offers an action based on the user's current state, and then receives the feedback from the user (e.g., next state and reward, etc.). This feedback is used by the agent to learn the unknown environment and improve its action selection strategy.

However, in most practical scenarios, the feedback from the users often encodes their sensitive information. Let us consider the user-agent interactions in Google Keyboard (Gboard) application. Here, each user is associated with a sequence of interactions with GBoard, e.g., the complete input of a sentence (or several sentences) can be viewed as an episode in the RL protocol. For each step in the episode, the state is the current typed words, and the action recommended by the GBoard agent is the most promising next words. The reward generated by the user depends on whether or not the user has adopted the offered next words as their actual input. It is easy to see that in this case each user's states and rewards contain her

*Wayne State University. Email: xingyu. zhou@wayne. edu 
sensitive information. Similarly, in a personalized healthcare setting, the states of a patient include personal information such as age, gender, height, weight, state of the treatment etc. Another intriguing example is the social robot for second language education of children (Gordon et al., 2016). The states include facial expressions, and the rewards contain whether they have passed the quiz. In all of these RL-based personalized applications, users may not want any of their sensitive information to be inferred by adversaries. This directly results in an increasing concern about privacy protection in nowadays personalized services. To be more specific, although a user might be willing to share her own information to the agent to obtain a better tailored service, she would not like to allow third parties to infer her private information from the output of the learning algorithm. For example, in the GBoard example, one would like to ensure that an adversary with arbitrary side knowledge and computation power cannot infer her sensitive information by looking at the recommended words to other users.

To this end, differential privacy (DP) (Dwork, 2008) has become a standard mechanism for designing interactive learning algorithms under a rigorous privacy guarantee for individual data. Most of the previous works on differentially private learning under partial feedback focus on the simpler bandit setting (i.e., no notion of states) (Tossou and Dimitrakakis, 2016, 2017; Basu et al., 2019; Mishra and Thakurta, 2015; Zhou and Tan, 2020; Shariff and Sheffet, 2018). For the general RL problem, there are only a few works that consider differential privacy (Vietri et al., 2020; Garcelon et al., 2020). More importantly, only the tabular MDPs are considered in these works where the states and actions are discrete and finite, and the value function is represented by a table. However, in real-world applications mentioned above, the number of states and actions are often very large and can even be infinite. As a result, the existing private RL algorithms could even fail and moreover their regret bounds (Vietri et al., 2020; Garcelon et al., 2020) become quite loose in this case since they are polynomial functions in terms of the number of states and actions. Thus, one fundamental question that remains elusive is: Is it possible to design private RL algorithms under large state and action spaces with tight regret guarantees? Furthermore, existing regret bounds in both (Vietri et al., 2020; Garcelon et al., 2020) are only for value-based RL algorithms, and a study on policy-based private RL algorithms remains open. Recently, policy optimization (PO) has seen great success in many real-world applications, especially when coupled with function approximations (Silver et al., 2017; Duan et al., 2016; Wang et al., 2018), and a variety of PO based algorithms have been proposed (Williams, 1992; Kakade, 2001; Schulman et al., 2015, 2017; Konda and Tsitsiklis, 2000). The theoretical understandings of PO have also been studied in both computational (i.e., convergence) perspective (Liu et al., 2019; Wang et al., 2019) and statistical (i.e., regret) perspective (Cai et al., 2020; Efroni et al., 2020). Unfortunately, all of these algorithm are non-private and thus a direct application of them on the above personalized services may lead to privacy concerns. The recent work (Chowdhury and Zhou, 2021) takes the first step towards private policy optimization in the tabular setting. Thus, another fundamental question is: Can we also design sample-efficient policy-based private RL algorithms with linear function approximations?

In this paper, we take a systematic approach to affirmatively answer the above two fundamental questions. In particular, our contributions can be summarized as follows.

- We take the first step towards designing privacy preserving RL algorithms with rigorous statistical guarantees (i.e., regrets) in MDPs with large state and action spaces. Specifically, under the linear function approximations (i.e., linear mixture MDPs), we design both value iteration and policy optimization based optimistic private RL algorithms and show that they enjoy sublinear regret in total number of steps while guaranteeing joint differential privacy (JDP), which, informally, implies that sensitive information about a given user is protected even if an adversary has access to the actions prescribed to all other users. 
- The regret bound of the value-based algorithm - Private-LinOpt-VI - is independent of the number of states and actions whereas the regret of the policy-based algorithm-Private-LinOpt-PO - depends at most logarithmically on the number of actions making them suitable for large scale MDPs. Moreover, the cost of privacy under $(\varepsilon, \delta)$-JDP for both Private-Linopt-VI and Private-LinOpt-PO scale only with $\frac{\log (1 / \delta)^{1 / 4}}{\varepsilon^{1 / 2}}$ given the permitted privacy budgets $\varepsilon>0$ and $\delta \in(0,1]$.

- The above results are achieved via a general procedure for learning in linear mixture MDPs under changing regularizers, which not only generalizes previous results for non-private learning for both valued-based and policy-based RL, but also serves as a building block for general private RL under various privacy schemes and different privacy guarantees. In particular, as opposed to the existing works, where same regularizers are used in every episode, our approach yields robust parameter estimates and confidence sets against possible adversarial attacks, paving the way for protecting data privacy.

\subsection{Related Work}

Beside the papers mentioned above, there are other related work on differentially private online learning (Guha Thakurta and Smith, 2013; Agarwal and Singh, 2017) and multi-armed bandits (Tossou and Dimitrakakis, 2017; Hu et al., 2021; Sajed and Sheffet, 2019). In the linear bandit setting with contextual information, (Shariff and Sheffet, 2018 ) shows an impossibility result, i.e., no algorithm can achieve a standard $(\varepsilon, \delta)$-DP privacy guarantee while guaranteeing a sublinear regret and thus the relaxed notion of JDP is considered in their paper. This result also implies that standard DP is incompatible with regret minimization in RL. Recently, another variant of DP, called local differential privacy (LDP) (Duchi et al., 2013) has gained increasing popularity in personalized services due to its stronger privacy protection. It has been studied in various bandit settings recently (Ren et al., 2020; Zheng et al., 2020; Zhou and Tan, 2020; Dubey, 2021). Under LDP, each user's raw data is directly protected before being sent to the learning agent. Thus, the learning agent only has access to privatized data to train its algorithm, which often leads to a worse regret guarantee compared to DP or JDP. Note that although our main focus is on private RL under JDP constraints, our proposed framework provides a straightforward way to design private RL algorithms under LDP constraints.

In the context of value-based tabular MDPs, (Vietri et al., 2020) gives the first regret bound under JDP while (Garcelon et al., 2020) presents the first regret bound under LDP constraint. As mentioned before, both bounds become loose in the presence of large state and action spaces. In addition to regret minimization, (Balle et al., 2016) considers private policy evaluation with linear function approximation. For MDPs with continuous state spaces, (Wang and Hegde, 2019) proposes a variant of $Q$-learning to protect only the rewards information by directly injecting noise into value functions. Recently, a distributed actor-critic RL algorithm under LDP is proposed in (Ono and Takahashi, 2020) but without any regret guarantee. While there are recent advances in regret guarantees for policy optimization (Cai et al., 2020; Efroni et al., 2020; Zhou et al., 2021a; He et al., 2021a), we are not aware of any existing work on private policy optimization.

Concurrent and independent work: There are two concurrent and independent works (Liao et al., 2021) and (Luyo et al., 2021) ${ }^{1}$. In particular, Liao et al. (2021) also studied differentially private reinforcement learning under linear mixture MDPs. In particular, they focused on local differential privacy (LDP) and proposed a private variant of UCRL-VTR (Ayoub et al., 2020). In contrast, we mainly focus on joint differential privacy (JDP) and investigated private variants for both value-based (i.e., UCRL-VTR) and policy-based

\footnotetext{
${ }^{1}$ While we upload to Arxiv after our acceptatnce, which is later than both works, we submit our work before Oct. 20, 2021, which is the fall deadline of ACM Sigmetrics conference.
} 
(i.e., OPPO) algorithms. As already highlighted in our paper, although our main focus is JDP, our established framework (relying a general REGULARIZER and general regret bounds) also provides a convenient way to obtain the regret bounds under LDP constraints as in (Liao et al., 2021), following the same modifications in our previous work (Chowdhury and Zhou, 2021) (see Remarks 6.9 and 6.6). The key difference compared to the JDP case is that now the PRIVATIZER is placed at each local user and in total $O(K)$ private noise rather than $O(\log K)$ is added since each PRIVATIZER at each user adds one independent noise. Nevertheless, for a direct and detailed treatment of privacy guarantee and regret bounds under LDP constraints, we refer readers to (Liao et al., 2021). Finally, in addition to a regret upper bound, (Liao et al., 2021) also presented a lower bound under LDP constraints.

In the concurrent work (Luyo et al., 2021), the authors also proposed a unified analysis for private UCRLVTR under both JDP and LDP constraints, which shares a similar high-level idea as ours but more detailed results on the LDP part is provided in (Luyo et al., 2021). While we further study private policy-based algorithms with linear function approximation, Luyo et al. (2021) further investigates private model-free algorithms and highlights interesting and unique issues in the model-free setting.

\section{Problem Formulation and Preliminaries}

In this section, we present the basics of episodic Markov decision processes with linear function approximations and introduce various notions of differential privacy in reinforcement learning.

\subsection{Learning Problem and Linear Function Approximations}

In this paper, we consider an episodic $\operatorname{MDP}\left(\mathcal{S}, \mathcal{A}, H,\left(\mathbb{P}_{h}\right)_{h=1}^{H},\left(r_{h}\right)_{h=1}^{H}\right)$, where $\mathcal{S}$ and $\mathcal{A}$ are state and action spaces, $H$ is the length of each episode, $\mathbb{P}_{h}\left(s^{\prime} \mid s, a\right)$ is the probability of transitioning to state $s^{\prime}$ from state $s$ provided action $a$ is taken at step $h$ and $r_{h}(s, a)$ is the mean of the reward distribution at step $h$ supported on $[0,1]$. The actions are chosen following some policy $\pi=\left(\pi_{h}\right)_{h=1}^{H}$, where each $\pi_{h}$ is a mapping from the state space $\mathcal{S}$ into a probability distribution over the action space $\mathcal{A}$, i.e. $\pi_{h}(a \mid s) \geq 0$ and $\sum_{a \in \mathcal{A}} \pi_{h}(a \mid s)=1$ for all $s \in \mathcal{S}$. The agent would like to find a policy $\pi$ that maximizes the long term expected reward starting from every state $s \in \mathcal{S}$ and every step $h \in[H]$, defined as

$$
V_{h}^{\pi}(s):=\mathbb{E}\left[\sum_{h^{\prime}=h}^{H} r_{h^{\prime}}\left(s_{h^{\prime}}, a_{h}^{\prime}\right) \mid s_{h}=s, \pi\right],
$$

where the expectation is with respect to the randomness of the transition kernel and the policy. We call $V_{h}^{\pi}$ the value function of policy $\pi$ at step $h$. Now, defining the $Q$-function of policy $\pi$ at step $h$ as

$$
\left.Q_{h}^{\pi}(s, a):=\mathbb{E}\left[\sum_{h^{\prime}=h}^{H} r_{h^{\prime}}\left(s_{h^{\prime}}, a_{h}^{\prime}\right)\right) \mid s_{h}=s, a_{h}=a, \pi\right],
$$

we obtain $Q_{h}^{\pi}(s, a)=r_{h}(s, a)+\sum_{s^{\prime} \in \mathcal{S}} V_{h+1}^{\pi}\left(s^{\prime}\right) P_{h}\left(s^{\prime} \mid s, a\right)$ and $V_{h}^{\pi}(s)=\sum_{a \in \mathcal{A}} Q_{h}^{\pi}(s, a) \pi_{h}(a \mid s)$.

A policy $\pi^{*}$ is said to be optimal if it maximizes the value for all states $s$ and step $h$ simultaneously, and the corresponding optimal value function is denoted by $V_{h}^{*}(s)=\max _{\pi \in \Pi} V_{h}^{\pi}(s)$ for all $h \in[H]$, where $\Pi$ is the set of all non-stationary policies. The agent interacts with the environment for $K$ episodes to learn the unknown transition probabilities $\mathbb{P}_{h}\left(s^{\prime} \mid s, a\right)$ and mean rewards $r_{h}(s, a)$, and thus, in turn, the optimal policy $\pi^{*}$. At each episode $k$, the agent chooses a policy $\pi^{k}=\left(\pi_{h}^{k}\right)_{h=1}^{H}$ and samples a trajectory $\left\{s_{1}^{k}, a_{1}^{k}, r_{1}^{k}, \ldots, s_{H}^{k}, a_{H}^{k}, r_{H}^{k}, s_{H+1}^{k}\right\}$ by interacting with the MDP using this policy. Here, at a given step $h$, $s_{h}^{k}$ denotes the state of the MDP, $a_{h}^{k} \sim \pi_{h}^{k}\left(\cdot \mid s_{h}^{k}\right)$ denotes the action taken by the agent, $r_{h}^{k} \in[0,1]$ denotes 
the (random) reward collected by the agent with the mean value $r_{h}\left(s_{h}^{k}, a_{h}^{k}\right)$ and $s_{h+1}^{k} \sim \mathbb{P}_{h}\left(\cdot \mid s_{h}^{k}, a_{h}^{k}\right)$ denotes the next state. The initial state $s_{1}^{k}$ is assumed to be fixed and history independent. We measure performance of the agent by the cumulative (pseudo) regret accumulated over $K$ episodes, defined as

$$
R(T):=\sum_{k=1}^{K}\left[V_{1}^{*}\left(s_{1}^{k}\right)-V_{1}^{\pi^{k}}\left(s_{1}^{k}\right)\right],
$$

where $T=K H$ denotes the total number of steps. We seek algorithms with regret that is sublinear in $T$, which demonstrates the agent's ability to act near optimally.

In this paper, we consider episodic MDPs with linear function approximations that allows us to work with large state and action spaces. Specifically, we consider the setting where both the transition dynamics and reward functions are linear with respect to some corresponding feature maps. This setting is often called linear mixture MDPs (a.k.a., linear kennel MDPs), see (Ayoub et al., 2020; Zhou et al., 2021b) for various examples of linear mixture MDPs including the tabular MDPs.

Assumption 1 (Linear mixture MDPs). We assume that the $\operatorname{MDP}\left(\mathcal{S}, \mathcal{A}, H,\left\{\mathbb{P}_{h}\right\}_{h=1}^{H},\left\{r_{h}\right\}_{h=1}^{H}\right)$ is a linear mixture MDP with a known transition feature mapping $\psi: \mathcal{S} \times \mathcal{A} \times \mathcal{S} \rightarrow \mathbb{R}^{d_{1}}$ and a known value feature mapping $\varphi: \mathcal{A} \times \mathcal{S} \rightarrow \mathbb{R}^{d_{2}}$. That is, for any $h \in[H]$, there exists an unknown vector $\theta_{h, p} \in \mathbb{R}^{d_{1}}$ with $\left\|\theta_{h, p}\right\|_{2} \leq \sqrt{d_{1}}$ such that

$$
\mathbb{P}\left(s^{\prime} \mid s, a\right)=\left\langle\psi\left(s, a, s^{\prime}\right), \theta_{h, p}\right\rangle
$$

for any $\left(s, a, s^{\prime}\right) \in \mathcal{S} \times \mathcal{A} \times \mathcal{S}$, and there exists an unknown vector $\theta_{h, r} \in \mathbb{R}^{d_{2}}$ with $\left\|\theta_{h, r}\right\|_{2} \leq \sqrt{d_{2}}$ such that

$$
r_{h}(s, a)=\left\langle\varphi(s, a), \theta_{h, r}\right\rangle
$$

for any $(s, a) \in \mathcal{S} \times \mathcal{A}$. Moreover, we assume $\|\varphi(s, a)\| \leq 1$ for any $(s, a) \in \mathcal{S} \times \mathcal{A}$ and let $\phi(s, a):=$ $\int_{\mathcal{s}^{\prime}} \psi\left(s^{\prime} \mid s, a\right) V\left(s^{\prime}\right) d s^{\prime}$ and assume that for any function $V: \mathcal{S} \rightarrow[0, H]\|\phi(s, a)\|_{2} \leq \sqrt{d_{1}} H$ for any $(s, a) \in \mathcal{S} \times \mathcal{A}$, and $\max \left\{d_{1}, d_{2}\right\} \leq d$.

The class of linear mixture MDPs has been adopted in a series of recent (non-private) RL works with linear function approximation including both value-based algorithms (Jia et al., 2020; Ayoub et al., 2020; Zhou et al., 2021b,a; He et al., 2021b) and policy-based algorithms (Cai et al., 2020; Ding et al., 2021). Another line of works with linear function approximation adopt linear MDP model (Jin et al., 2020; Yang and Wang, 2019), which assumes that $\mathbb{P}(\cdot \mid s, a)=\langle\tau(s, a), \mu(\cdot)\rangle$ where $\tau(s, a)$ is a known feature mapping and $\mu(\cdot)$ is an unknown measure. Note that the two commonly used linear models are different and one cannot be recovered by the other, see a detailed discussions in (Zhou et al., 2021b).

\subsection{Differential Privacy in Episodic RL}

In RL-based personalized services, it is common to view each episode $k \in[K]$ as a trajectory associated to a specific user (cf. Google GBoard application). To this end, we let $U_{K}=\left(u_{1}, \ldots, u_{K}\right) \in \mathcal{U}^{K}$ to denote a sequence of $K$ unique $^{2}$ users participating in the private RL protocol with an RL agent $\mathcal{M}$, where $\mathcal{U}$ is the set of all users. Formally speaking, as in (Vietri et al., 2020; Garcelon et al., 2020), a user can be captured with a tree of depth $H$ encoding the state and reward responses she would give to all the $A^{H}$ possible sequences

\footnotetext{
${ }^{2}$ Uniqueness is assumed for simplicity, as for a returning user one can group her with her previous occurrences and use the notion of group privacy to provide tight privacy guarantee.
} 
of actions the agent can choose. During the learning process, user $k$ for episode $k$ is identified by the reward and state responses $\left(r_{h}^{k}, s_{h+1}^{k}\right)_{h=1}^{H}$ she gives to the actions $\left(a_{h}^{k}\right)_{h=1}^{H}$ chosen by the agent under policy $\pi^{k}$. We let $\mathcal{M}\left(U_{k}\right)=\left(a_{1}^{1}, \ldots, a_{H}^{K}\right) \in \mathcal{A}^{K H}$ to denote the set of all actions chosen by the agent $\mathcal{M}$ when interacting with the user sequence $U_{k}$. Informally, we will be interested in (centralized) randomized mechanisms (in this case, RL agents) $\mathcal{M}$ so that the knowledge of the output $\mathcal{M}\left(U_{k}\right)$ and all but the $k$-th user $u_{k}$ does not reveal 'much' information about $u_{k}$. We formalize this in the following definition.

Definition 2.1 (Differential Privacy (DP)). For any $\varepsilon \geq 0$ and $\delta \in[0,1]$, a mechanism $\mathcal{M}: \mathcal{U}^{K} \rightarrow \mathcal{A}^{K H}$ is $(\varepsilon, \delta)$-differentially private if for all $U_{K}, U_{K}^{\prime} \in \mathcal{U}^{K}$ differing on a single user and for all subset of actions $\mathcal{A}_{0} \subset \mathcal{A}^{K H}$,

$$
\mathbb{P}\left[\mathcal{M}\left(U_{K}\right) \in \mathcal{A}_{0}\right] \leq \exp (\varepsilon) \mathbb{P}\left[\mathcal{M}\left(U_{K}^{\prime}\right) \in \mathcal{A}_{0}\right]+\delta .
$$

This is a direct adaptation of the classic notion of differential privacy (Dwork et al., 2014). However, we need to relax this definition for our purpose, because standard DP requires that changing the states (or reward response) of a user, the recommended actions to this user will not change too much, which is in conflict with personalization. In fact, even in the simpler linear contextual bandit setting, a negative result says that no algorithm can achieve a sublinear regret while guaranteeing standard DP (Shariff and Sheffet, 2018). Hence, to preserve the privacy of individual users while obtaining a nontrivial regret in our RL setting, as in previous works (Shariff and Sheffet, 2018; Vietri et al., 2020), we consider the notion of joint differential privacy (JDP) (Kearns et al., 2014), which requires that simultaneously for all user $u_{k}$, the joint distribution of the actions recommended to all users other than $u_{k}$ be differentially private with respect to the user $u_{k}$. It weakens the constraint of DP only in that the actions suggested specifically to $u_{k}$ may be sensitive in her type (state and reward responses), which is indeed the intrinsic requirement of personalization services. However, JDP is still a very strong definition since it protects $u_{k}$ from any arbitrary collusion of other users against her, so long as she does not herself make the actions suggested to her public. To this end, we let $\mathcal{M}_{-k}\left(U_{K}\right):=\mathcal{M}\left(U_{K}\right) \backslash\left(a_{h}^{k}\right)_{h=1}^{H}$ to denote all the actions chosen by the agent $\mathcal{M}$ excluding those recommended to $u_{k}$ and formally define JDP as follows.

Definition 2.2 (Joint Differential Privacy (JDP)). For any $\varepsilon \geq 0$ and $\delta \in[0,1]$, a mechanism $\mathcal{M}: \mathcal{U}^{K} \rightarrow \mathcal{A}^{K H}$ is $(\varepsilon, \delta)$-joint differentially private if for all $k \in[K]$, for all user sequences $U_{K}, U_{K}^{\prime} \in \mathcal{U}^{K}$ differing only on the $k$-th user and for all set of actions $\mathcal{A}_{-k} \subset \mathcal{A}^{(K-1) H}$ given to all but the $k$-th user,

$$
\mathbb{P}\left[\mathcal{M}_{-k}\left(U_{K}\right) \in \mathcal{A}_{-k}\right] \leq \exp (\varepsilon) \mathbb{P}\left[\mathcal{M}_{-k}\left(U_{K}^{\prime}\right) \in \mathcal{A}_{-k}\right]+\delta .
$$

Intuitively speaking, JDP in RL means that upon changing one of the participating users (say user $k$ ), the information observed by the other $K-1$ participants will not change substantially. JDP has been used extensively in private mechanism design (Kearns et al., 2014), in private matching and allocation problems (Hsu et al., 2016), in designing privacy-preserving algorithms for linear contextual bandits (Shariff and Sheffet, 2018), linear quadratic systems (Chowdhury et al., 2021) and it has been introduced in private tabular RL by Vietri et al. (2020).

In this work, we mainly focus on algorithms that are $(\varepsilon, \delta)$-JDP to illustrate the power of our proposed general framework for private RL algorithms. However, in addition to JDP guarantees, our framework also easily carries out to RL with stringer notion of privacy protection, called the local differential privacy (LDP) (Duchi et al., 2013), which is often applied to scenarios where the users may not even be willing to share it's data with the agent directly. In this setting, each user is assumed to have her own privacy mechanism that can do randomized mapping on its data to guarantee privacy. To this end, we denote by $X$ a trajectory $\left(s_{h}, a_{h}, r_{h}, s_{h+1}\right)_{h=1}^{H}$ and by $\mathcal{X}$ the set of all possible trajectories. We write $\mathcal{M}^{\prime}(X)$ to denote 
the privatized trajectory generated by a (local) randomized mechanism $\mathcal{M}^{\prime}$. With this notation, we now formally define LDP for RL protocol.

Definition 2.3 (Local Differential Privacy (LDP)). For any $\varepsilon \geq 0$ and $\delta \in[0,1]$, a mechanism $\mathcal{M}^{\prime}$ is $(\varepsilon, \delta)$ local differentially private if for all trajectories $X, X^{\prime} \in \mathcal{X}$ and for all possible subsets $\mathcal{E}_{0} \subset\left\{\mathcal{M}^{\prime}(X) \mid X \in\right.$ $\mathcal{X}\}$,

$$
\mathbb{P}\left[\mathcal{M}^{\prime}(X) \in \mathcal{E}_{0}\right] \leq \exp (\varepsilon) \mathbb{P}\left[\mathcal{M}^{\prime}\left(X^{\prime}\right) \in \mathcal{E}_{0}\right]+\delta .
$$

LDP ensures that if any adversary (can be the RL agent itself) observes the output of the privacy mechanism $\mathcal{M}^{\prime}$ for two different trajectories, then it is statistically difficult for it to guess which output is from which trajectory. This has been used extensively in multi-armed bandits (Zheng et al., 2020; Ren et al., 2020), and introduced in private tabular RL by Garcelon et al. (2020).

\section{Confidence Bounds with Changing Regularizers}

In this section, we focus on the key component of both algorithm design and regret analysis under linear mixture MDPs for both value-based and policy-based algorithms. That is, the confidence bounds over the regularized least-squared estimators for unknown $\theta_{h, p}$ and $\theta_{h, r}$. In particular, we consider a general setting with changing regularizers, which not only allows us to generalize previous non-private ones, but more importantly servers a general procedure for designing private RL algorithms with linear function approximations in later sections.

To begin with, let's briefly introduce the intuitions behind the regularized linear regression problem under linear mixture MDPs (cf. (Ayoub et al., 2020; Zhou et al., 2021a)). In particular, for any step $h$ at episode $k$, the following equation holds based on the linear mixture MDP model assumption in Assumption 1

$$
\left[\mathbb{P}_{h} V_{h+1}^{k}\right]\left(s_{h}^{k}, a_{h}^{k}\right)=\left\langle\int_{s^{\prime}} \psi\left(s^{\prime} \mid s_{h}^{k}, a_{h}^{k}\right) V_{h+1}^{k}\left(s^{\prime}\right) d s^{\prime}, \theta_{h, p}\right\rangle=\left\langle\phi_{h}^{k}\left(s_{h}^{k}, a_{h}^{k}\right), \theta_{h, p}\right\rangle,
$$

where $\left\{V_{h}^{k}(\cdot)\right\}_{(k, h) \in[K] \times[H]}$ is any value function maintained based on data collected before episode $k$, and $\phi_{h}^{k}(\cdot, \cdot):=\int_{s^{\prime}} \psi\left(s^{\prime} \mid \cdot, \cdot\right) V_{h+1}^{k}\left(s^{\prime}\right) d s^{\prime}$. Therefore, learning the true $\theta_{h, p}$ could be translated into solving a 'linear bandit' problem where the feature is $\phi_{h}^{k}\left(s_{h}^{k}, a_{h}^{k}\right)$ and the noise is $V_{h+1}^{k}\left(s_{h+1}^{k}\right)-\left[\mathbb{P}_{h} V_{h+1}^{k}\right]\left(s_{h}^{k}, a_{h}^{k}\right)$. To this end, one can solve the following regularized linear regression problem:

$$
\begin{aligned}
& \theta_{h, p}^{k}:=\underset{\theta \in \mathbb{R}^{d_{1}}}{\operatorname{argmin}}\|\theta\|_{Z_{h, p}^{k}}+\sum_{j=1}^{k-1}\left[V_{h+1}^{j}\left(s_{h+1}^{j}\right)-\left\langle\phi_{h}^{k}\left(s_{h}^{j}, a_{h}^{j}\right), \theta\right\rangle\right]^{2} \\
& =\underbrace{\left[Z_{h, p}^{k}+\sum_{j=1}^{k-1} \phi_{h}^{k}\left(s_{h}^{j}, a_{h}^{j}\right) \phi_{h}^{k}\left(s_{h}^{j}, a_{h}^{j}\right)^{\top}\right.}_{\mathcal{T}_{1, p}}]^{-1}[\underbrace{\sum_{j=1}^{k-1} \phi_{h}^{k}\left(s_{h}^{j}, a_{h}^{j}\right) V_{h+1}^{j}\left(s_{h+1}^{j}\right)}_{\mathcal{T}_{2, p}}],
\end{aligned}
$$

where $Z_{h, p}^{k} \in \mathbb{R}^{d_{1} \times d_{1}}$ is possibly changing positive definite matrix. Setting $\Lambda_{h, p}^{k}=\mathcal{T}_{1, p}$ and $u_{h, p}^{k}=\mathcal{T}_{2, p}+$ $z_{h, p}^{k}$ for some $z_{h, p}^{k} \in \mathbb{R}^{d_{1}}$, we can define a new estimator $\widehat{\theta}_{h, p}^{k}$ under changing regularizers $\left\{Z_{h, p}^{k}\right\}_{k \in[K], h \in[H]}$ and $\left\{z_{h, p}^{k}\right\}_{k \in[K], h \in[H]}$ as

$$
\widehat{\theta}_{h, p}^{k}=\left[\Lambda_{h, p}^{k}\right]^{-1} u_{h, p}^{k}
$$


In particular, choosing $Z_{h, p}^{k}=\lambda I$ and $z_{h, p}^{k}=0$ for all $h, k$ reduces to the standard non-private value-based algorithms (Ayoub et al., 2020; Zhou et al., 2021a; Cai et al., 2020) and policy-based algorithm (Cai et al., 2020; Ding et al., 2021) under linear mixture MDPs. Here, $Z_{h, p}^{k}$ serves as a general regularizer while $z_{h, p}^{k}$ can be used to account for the noisy version of $\mathcal{T}_{2, p}$ where the noise could arise from approximation error of $\phi_{h}^{k}$, or, from the added Gaussian noise in DP mechanisms in later sections.

In this paper, we also consider a random reward as opposed to a deterministic reward in previous works. To this end, for the estimator of $\theta_{h, r}$, we consider the minimizer of the following linear regression problem with a positive definite matrix $Z_{h, r}^{k}$.

$$
\begin{aligned}
\theta_{h, r}^{k} & :=\underset{\theta \in \mathbb{R}^{d_{2}}}{\operatorname{argmin}}\|\theta\|_{Z_{h, r}^{k}}+\sum_{j=1}^{k-1}\left[r_{h}^{j}\left(s_{h}^{j}, a_{h}^{j}\right)-\left\langle\varphi\left(s_{h}^{j}, a_{h}^{j}\right), \theta\right\rangle\right]^{2} \\
& =\underbrace{\left[Z_{h, r}^{k}+\sum_{j=1}^{k-1} \varphi\left(s_{h}^{j}, a_{h}^{j}\right) \varphi\left(s_{h}^{j}, a_{h}^{j}\right)^{\top}\right.}_{\mathcal{T}_{1, r}}]^{-1}[\underbrace{\left.\sum_{j=1}^{k-1} \varphi\left(s_{h}^{j}, a_{h}^{j}\right) r_{h}^{j}\left(s_{h}^{j}, a_{h}^{j}\right)\right]}_{\mathcal{T}_{2, r}} .
\end{aligned}
$$

Setting $\Lambda_{h, r}^{k}=\mathcal{T}_{1, r}$ and $u_{h, r}^{k}=\mathcal{T}_{2, r}+z_{h, r}^{k}$ for some $z_{h, r}^{k} \in \mathbb{R}^{d_{2}}$, we can define a new estimator $\widehat{\theta}_{h, r}^{k}$ under changing regularizers $\left\{Z_{h, r}^{k}\right\}_{k \in[K], h \in[H]}$ and $\left\{z_{h, r}^{k}\right\}_{k \in[K], h \in[H]}$ as

$$
\widehat{\theta}_{h, r}^{k}=\left[\Lambda_{h, r}^{k}\right]^{-1} u_{h, r}^{k}
$$

One key question now is to guarantee that the two estimators $\widehat{\theta}_{h, p}^{k}$ and $\widehat{\theta}_{h, r}^{k}$ are close to the true parameters $\theta_{h, p}$ and $\theta_{h, r}$ with properly constructed ellipsoids. That is, even under changing regularizers, both $\theta_{h, p}$ and $\theta_{h, r}$ lie in the following ellipsoids with probability at least $1-\alpha$ for any $\alpha \in(0,1]$, respectively

$$
\mathcal{C}_{h, p}^{k}=\left\{\theta:\left\|\theta-\widehat{\theta}_{h, p}^{k}\right\|_{\Lambda_{h, p}^{k}} \leq \beta_{p}(\alpha)\right\} \text { and } \mathcal{C}_{h, r}^{k}=\left\{\theta:\left\|\theta-\widehat{\theta}_{h, r}^{k}\right\|_{\Lambda_{h, r}^{k}} \leq \beta_{r}(\alpha)\right\}
$$

where $\beta_{p}(\alpha)$ and $\beta_{r}(\alpha)$ are properly chosen parameters to balance between exploration and exploitation. Throughout this paper, we will make the following assumptions on the sequence of regularizers $\left\{Z_{h, p}^{k}\right\}_{k \in[K], h \in[H]}$, $\left\{z_{h, p}^{k}\right\}_{k \in[K], h \in[H]},\left\{Z_{h, r}^{k}\right\}_{k \in[K], h \in[H]}$ and $\left\{z_{h, r}^{k}\right\}_{k \in[K], h \in[H]}$.

Assumption 2 (Regularity). For any $\alpha \in(0,1]$, there exist constants $\lambda_{\max , p}, \lambda_{\max , r}, \lambda_{\min , p}, \lambda_{\min , r}$ and $\nu_{p}, \nu_{r}$ depending on $\alpha$ such that, with probability at least $1-\alpha$, for all $k \in[K]$ and $h \in[H]$, the following relations hold simultaneously

$$
\left\|Z_{h, p}^{k}\right\| \leqslant \lambda_{\max , p}, \quad\left\|\left(Z_{h, p}^{k}\right)^{-1}\right\| \leqslant 1 / \lambda_{\min , p} \text { and }\left\|z_{h, p}^{k}\right\|_{\left(Z_{h, p}^{k}\right)^{-1}} \leqslant \nu_{p},
$$

and

$$
\left\|Z_{h, r}^{k}\right\| \leqslant \lambda_{\max , r}, \quad\left\|\left(Z_{h, r}^{k}\right)^{-1}\right\| \leqslant 1 / \lambda_{\min , r} \text { and }\left\|z_{h, r}^{k}\right\|_{\left(Z_{h, r}^{k}\right)^{-1}} \leqslant \nu_{r} .
$$

With this regularity assumption, we have the following high-probability confidence bounds over the two estimators, i.e., $\widehat{\theta}_{h, p}^{k}$ in (1) and $\widehat{\theta}_{h, r}^{k}$ in (2). 
Lemma 3.1 (Concentration under changing regularizers). Let Assumptions 1 and 2 hold and $V_{h}^{k}(\cdot)$ in (1) be within $[0, H]$ for all $h \in[H], k \in[K]$. Then, for any $\alpha \in(0,1]$, with probability at least $1-3 \alpha$, the following relations hold simultaneously for all $k \in[K]$ and $h \in[H]$

$$
\left\|\theta_{h, p}-\widehat{\theta}_{h, p}^{k}\right\|_{\Lambda_{h, p}^{k}} \leq \beta_{p}(\alpha), \text { and }\left\|\theta_{h, r}-\widehat{\theta}_{h, r}^{k}\right\|_{\Lambda_{h, r}^{k}} \leq \beta_{r}(\alpha),
$$

where

$$
\begin{aligned}
& \beta_{p}(\alpha)=\frac{H}{2} \sqrt{2 \ln \left(\frac{H}{\alpha}\right)+d_{1} \ln \left(1+\frac{K H^{2}}{\lambda_{\min , p}}\right)}+\sqrt{d_{1} \lambda_{\max , p}}+\nu_{p}, \\
& \beta_{r}(\alpha)=\frac{1}{2} \sqrt{2 \ln \left(\frac{H}{\alpha}\right)+d_{2} \ln \left(1+\frac{K}{d_{2} \lambda_{\min , r}}\right)}+\sqrt{d_{2} \lambda_{\max , r}}+\nu_{r} .
\end{aligned}
$$

Proof. See Appendix A.

In the next two sections, we will utilize the two confidence bounds to investigate general value-based and policy-based RL algorithms under changing regularizers. These results not only generalize the standard results for fixed regularizers, but allow us to present a general framework for designing private RL algorithms with differential privacy guarantees in Section 6.

\section{Optimistic Value-based Algorithm with Changing Regularizers}

In this section, we propose an optimistic value-based RL algorithm under linear mixture MDPs with changing regularizers. Our algorithm can be regarded as a generalization of UCRL-VTR (Ayoub et al., 2020) where fixed regularizers are adopted. This generalization serves a basis for designing private value-based algorithm in Section 6.

As shown in Algorithm 1, compared to UCRL-VTR, the main difference of our LinOpt-VI-Reg is the adoption of the REGULARIZER that properly chooses the four sequences of regularizers $\left\{Z_{h, p}^{k}\right\}_{k \in[K], h \in[H]}$, $\left\{z_{h, p}^{k}\right\}_{k \in[K], h \in[H]},\left\{Z_{h, r}^{k}\right\}_{k \in[K], h \in[H]}$ and $\left\{z_{h, r}^{k}\right\}_{k \in[K], h \in[H]}$. Similar to UCRL-VTR, LinOpt-VI-Reg follows the procedure of optimistic value iteration. Specifically, at each episode $k$, it first computes the two estimators and the corresponding exploration bonus terms based on regularized statistics. Then, it computes the estimated $Q$-function and value function using optimistic Bellman recursion. Next, a greedy policy $\pi^{k}$ is obtained directly from the estimated $Q$-function. Finally, a trajectory is rolled out by acting the policy $\pi^{k}$ and then the REgULARIZER translates these statistics into regularized ones via the way in Section 3, i.e., using the four regularizers $\left\{Z_{h, p}^{k}\right\}_{k \in[K], h \in[H]},\left\{z_{h, p}^{k}\right\}_{k \in[K], h \in[H]},\left\{Z_{h, r}^{k}\right\}_{k \in[K], h \in[H]}$ and $\left\{z_{h, r}^{k}\right\}_{k \in[K], h \in[H]}$. The following theorem presents a general regret bound when the REGULARIZER in LinOpt-VI-Reg satisfies Assumption 2.

Theorem 4.1. Let Assumption 1 hold and the REgUlARIZER in Algorithm 1 satisfy Assumption 2. Then, for any $\alpha \in(0,1]$, with probability at least $1-4 \alpha$, Algorithm 1 achieves regret

$$
\mathcal{R}(T)=O\left(\sqrt{T H^{3}}\left(\ln \frac{H}{\alpha}+d \kappa_{\lambda_{\min }}\right)+\sqrt{T H d \kappa_{\lambda_{\min }}}\left(\sqrt{d \lambda_{\max }}+\nu\right)\right)
$$

where $\kappa_{\lambda_{\min }}:=\ln \left(1+K H^{2} /\left(\lambda_{\min }\right)\right)$ and $\lambda_{\min }=\min \left\{\lambda_{\min , p}, \lambda_{\min , r}\right\}, \lambda_{\max }=\max \left\{\lambda_{\max , p}, \lambda_{\max , r}\right\}$ and $\nu=\max \left\{\nu_{p}, \nu_{r}\right\}$ 


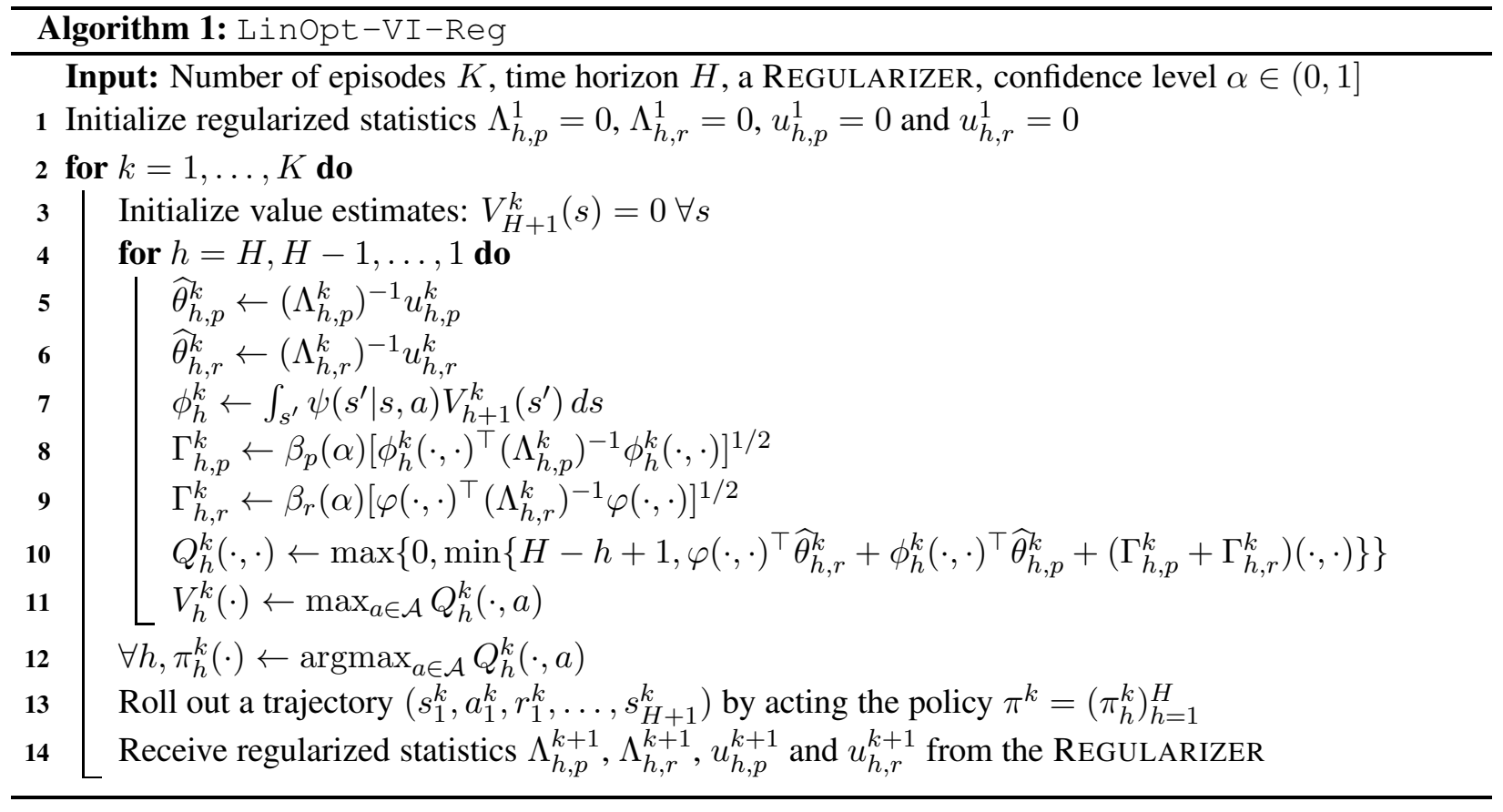

Proof Sketch. Let $\mathcal{E}(\alpha):=\left\{\theta_{h, p} \in \mathcal{C}_{h, p}^{k}\right.$ and $\left.\theta_{h, r} \in \mathcal{C}_{h, r}^{k}, \forall h \in[H], k \in[K]\right\}$ denote the 'good' event, which by Lemma 3.1, holds with probability at least $1-3 \alpha$. Under this good event, we can first show that $Q_{h}^{k}$ maintained in Algorithm 1 is optimistic with respect to the optimal $Q$-value $Q_{h}^{*}$. With this property and the linear mixture MDPs assumption, the regret can be decomposed as follows.

$$
\begin{aligned}
\sum_{k=1}^{K} V_{k, 1}\left(s_{1}^{k}\right)-V_{1}^{\pi^{k}}\left(s_{1}^{k}\right) & \leq \underbrace{\sum_{k=1}^{K} \sum_{h=1}^{H} 2 \beta_{p}(\alpha) \min \left\{1,\left\|\phi_{h}^{k}\left(s_{h}^{k}, a_{h}^{k}\right)\right\|_{\left(\Lambda_{h, p}^{k}\right)^{-1}}\right\}}_{\mathcal{T}_{1}} \\
& +\underbrace{\sum_{k=1}^{K} \sum_{h=1}^{H} 2 H \beta_{r}(\alpha) \min \left\{1,\left\|\varphi\left(s_{h}^{k}, a_{h}^{k}\right)\right\|_{\left.\left(\Lambda_{h, r}^{k}\right)^{-1}\right\}}\right\}}_{\mathcal{T}_{2}} \\
& +\underbrace{\sum_{k=1}^{K} \sum_{h=1}^{H} \mathbb{P}_{h}\left[V_{h+1}^{k}-V_{h}^{\pi^{k}}\right]\left(s_{h}^{k}, a_{h}^{k}\right)-\left[V_{h+1}^{k}-V_{h}^{\pi^{k}}\right]\left(s_{h+1}^{k}\right)}_{\mathcal{T}_{3}} .
\end{aligned}
$$

With a properly defined filtration, $\mathcal{T}_{3}$ is a sum of bounded martingale difference sequence. Therefore, by Azuma-Hoeffding inequality, we have with probability at least $1-\alpha, \mathcal{T}_{3} \leq H \sqrt{2 K H \log (1 / \alpha)}$, where we have used boundedenss of value function in Algorithm 1. To bound $\mathcal{T}_{1}$ and $\mathcal{T}_{2}$, we will use the tracedeterminant lemma and elliptical potential lemma (cf. (Abbasi-Yadkori et al., 2011, Lemmas 10 and 11)). In particular, by the boundedness of $\left\|\phi_{h}^{k}\left(s_{h}^{k}, a_{h}^{k}\right)\right\| \leq \sqrt{d_{1}} H$ and $\left\|\varphi\left(s_{h}^{k}, a_{h}^{k}\right)\right\| \leq 1$, and the Assumption 2 
on the REGULARIZER, we obtain that

$$
\begin{aligned}
& \mathcal{T}_{1} \leq 2 \beta_{p}(\alpha) \sqrt{K H} \sqrt{2 H d_{1} \log \left(1+K H^{2} /\left(\lambda_{\min , p}\right)\right)} \\
& \mathcal{T}_{2} \leq 2 H \beta_{r}(\alpha) \sqrt{K H} \sqrt{2 H d_{2} \log \left(1+K /\left(d_{2} \lambda_{\min , r}\right)\right)} .
\end{aligned}
$$

Finally, plugging in the choices of $\beta_{p}(\alpha)$ and $\beta_{r}(\alpha)$ in Lemma 3.1 and putting everything together, yields the required result. The full proof is given at Section 7.1.

Remark 4.2. This theorem serves as a building block for analyzing private value-based RL algorithm under linear mixture MDPs. In particular, the general procedure is to first instantiate the REGULARIZER with a specific privacy protection scheme, and then determine the corresponding values of $\lambda_{\max }, \lambda_{\min }, \nu$. Finally, with Theorem 4.1, one can easily obtain the private regret. One key feature of this result is that it provides a general analytical framework for private value-based algorithms under different private schemes and even different types of privacy guarantees (e.g., JDP and LDP). See more discussions in Section 6.

\section{Optimistic Policy-based Algorithm with Changing Regularizers}

In this section, we turn to focus on policy-based algorithm under linear mixture MDPs. Our proposed algorithm can be regarded as a bandit-feedback variant of the OPPO algorithm in (Cai et al., 2020) where the full information of the reward is observed and the regularizer is fixed. As in the last section, this generalization serves as a basis for designing private policy-based algorithm in later section.

As shown in Algorithm 2, our proposed LioOpt-PO-Reg basically consists of two stages at each episode $k$ : policy evaluation and policy improvement. In the policy evaluation stage, it uses the LeastSquares Temporal Difference (LSTD) (Boyan, 1999; Bradtke and Barto, 1996) to evaluate policy $\pi^{k}$ based on previous $k-1$ historical trajectories. In contrast to standard process, LinOpt-PO-Reg uses regualrized statistics produced by possible changing regularizers at this stage. In particular, it estimates $\mathbb{P}_{h} V_{h+1}^{k}$ with $\left(\phi_{h}^{k}\right)^{\top} \widehat{\theta}_{h, p}^{k}$ and estimates $r_{h}$ with $\varphi^{\top} \widehat{\theta}_{h, r}^{k}$. These two estimates along with two UCB exploration bonuses are used to iteratively compute $Q$-function estimates. The $Q$-estimates are then truncated to the interval $[0, H]$ since the reward is within $[0,1]$. Then, the corresponding value estimates are computed by taking the expectation of $Q$-estimates with respect to the policy. Next, a new trajectory is rolled out by acting the policy $\pi^{k}$ and the REGULARIZER translates the collected statistics into regularized ones via the four changing regularizers. Finally, in the policy improvement stage, LinOpt-PO-Reg employs a 'soft' update of the current policy $\pi^{k}$ by following a standard mirror-descent step together with a Kullback-Leibler (KL) divergence proximity term (Cai et al., 2020). The following theorem presents a general regret bound when the Regularizer in Linopt-PO-Reg satisfies Assumption 2.

Theorem 5.1. Let Assumption 1 hold and the REgUlARIZER in Algorithm 2 satisfy Assumption 2. Then, for any $\alpha \in(0,1]$, with probability at least $1-4 \alpha$, Algorithm 2 achieves regret

$$
\mathcal{R}(T)=O\left(\sqrt{T H^{3}}\left(\log \frac{H}{\alpha}+d \kappa_{\lambda_{\min }}\right)+\sqrt{T H^{3} \log |\mathcal{A}|}+\sqrt{T H d \kappa_{\lambda_{\min }}}\left(\sqrt{d \lambda_{\max }}+\nu\right)\right)
$$

where $\kappa_{\lambda_{\min }}:=\ln \left(1+K H^{2} /\left(\lambda_{\min }\right)\right)$ and $\lambda_{\min }=\min \left\{\lambda_{\min , p}, \lambda_{\min , r}\right\}, \lambda_{\max }=\max \left\{\lambda_{\max , p}, \lambda_{\max , r}\right\}$ and $\nu=\max \left\{\nu_{p}, \nu_{r}\right\}$. Further, suppose $\sqrt{\log |\mathcal{A}|}=O\left(\log \frac{H}{\alpha}+d \kappa_{\lambda_{\min }}\right)$, the regret satisfies

$$
\mathcal{R}(T)=O\left(\sqrt{T H^{3}}\left(\log \frac{H}{\alpha}+d \kappa_{\lambda_{\min }}\right)+\sqrt{T H d \kappa_{\lambda_{\min }}}\left(\sqrt{d \lambda_{\max }}+\nu\right)\right) .
$$


Proof Sketch. As in (Cai et al., 2020), our regret analysis starts with the following regret decomposition.

$$
\begin{aligned}
\mathcal{R}(T) & =\sum_{k=1}^{K}\left(V_{1}^{*}\left(s_{1}^{k}\right)-V_{1}^{\pi_{k}}\left(s_{1}^{k}\right)\right) \\
& =\underbrace{\sum_{k=1}^{K} \sum_{h=1}^{H} \mathbb{E}_{\pi^{*}}\left[\left\langle Q_{h}^{k}\left(s_{h}^{k}\right), \pi_{h}^{*}\left(\cdot \mid s_{h}\right)-\pi_{h}^{\pi_{k}}\left(\cdot \mid s_{h}\right)\right\rangle \mid s_{1}=s_{1}^{k}\right]}_{(i)}+\underbrace{\mathcal{M}_{K, H, 2}}_{(i i)} \\
& +\underbrace{\sum_{k=1}^{K} \sum_{h=1}^{H}\left(\mathbb{E}_{\pi^{*}}\left[\iota_{h}^{k}\left(x_{h}, a_{h}\right) \mid s_{1}=s_{1}^{k}\right]-\iota_{h}^{k}\left(s_{h}^{k}, a_{h}^{k}\right)\right)}_{(i i i)},
\end{aligned}
$$

where $\iota_{h}^{k}:=r_{h}+\mathbb{P}_{h} V_{h+1}^{k}-Q_{h}^{k}$ and $\mathcal{M}_{K, H, 2}$ is a martingale adapted to a properly defined filtration. Hence, by the boundedness of both $Q$-function and value function and Azuma-Hoeffding inequality, we can establish that with probability at least $1-\alpha,(i i) \leq 4 H \sqrt{2 T \log (1 / \alpha)}$. For $(i)$, we can bound it by using standard properties of KL-divergence and mirror-descent update along with the fact that $\pi^{1}$ is a uniform distribution. In particular, we have $(i) \leq \sqrt{2 H^{3} T \log |\mathcal{A}|}$. The key part in the proof is to bound (iii). To this end, we will first show that under good event $\mathcal{E}(\alpha)$, we have for all $s, a, k, h$,

$$
2\left(\Gamma_{h, p}^{k}+\Gamma_{h, r}^{k}\right)(s, a) \geq-\iota_{h}^{k}(s, a) \geq 0 .
$$

Therefore, with the above property, we can see that bounding (iii) reduces to bounding the summation of both bonus terms. Similar to the analysis in the last section, we have

$$
(i i i)=O\left(\sqrt{T H^{3}}\left(\log \frac{H}{\alpha}+d \kappa_{\lambda_{\min }}\right)+\sqrt{T H d \kappa_{\lambda_{\min }}}\left(\sqrt{d \lambda_{\max }}+\nu\right)\right),
$$

where $\kappa_{\lambda_{\min }}:=\log \left(1+K H^{2} /\left(\lambda_{\min }\right)\right)$ and $\lambda_{\min }=\min \left\{\lambda_{\min , p}, \lambda_{\min , r}\right\}, \lambda_{\max }=\max \left\{\lambda_{\max , p}, \lambda_{\max , r}\right\}$ and $\nu=\max \left\{\nu_{p}, \nu_{r}\right\}$. Finally, putting the three terms together, yields the required result. The full proof is given at Section 8.

Remark 5.2. This theorem provides a clean result to design the first private $P O$ algorithms with linear function approximations in the next section. As in the last section, this result offers a simple approach to designing private PO algorithms under different privacy guarantees, which will be explored in the next section.

\section{Privacy and Regret Guarantees}

In this section, we shall see how to instantiate the REGULARIZER in previous two algorithms with a specific privacy protection scheme-called PRIVATIZER hereafter-to guarantee JDP while achieving sublinear regret.

\subsection{Privatizer Design}

The main idea behind the PRIVATIZER is to inject Gaussian noise as the changing regularizers, i.e., $Z_{h, p}^{k}$ and $Z_{h, r}^{k}$ are chosen to be Gaussian random matrices and $z_{h, p}^{k}$ and $z_{h, r}^{k}$ are Gaussian random vectors so that 


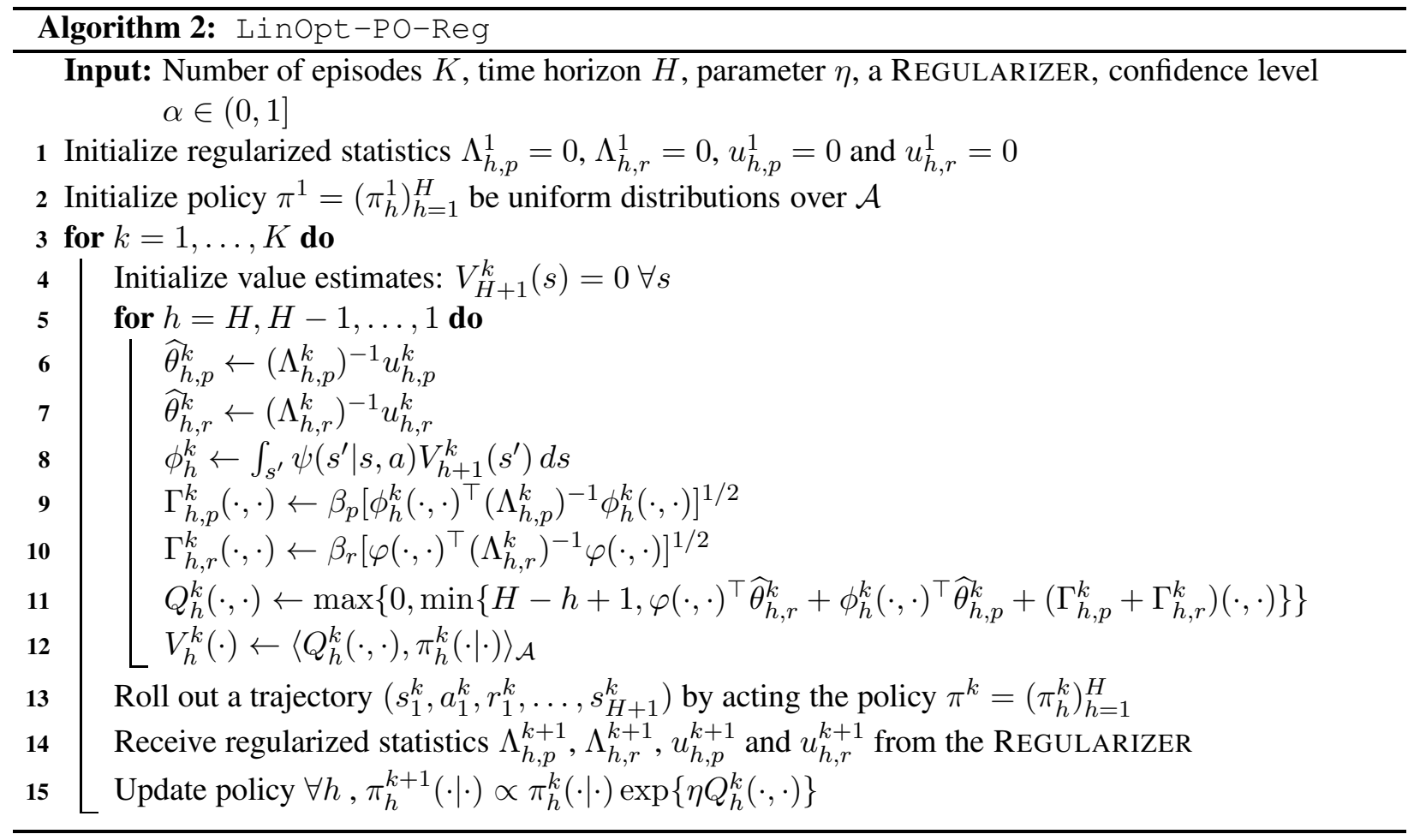

the standard Gaussian mechanism (Dwork et al., 2014) can be applied. These noise are used to privatize the non-private statistics $G_{h, p}^{k}:=\sum_{j=1}^{k-1} \phi_{h}^{k}\left(s_{h}^{j}, a_{h}^{j}\right) \phi_{h}^{k}\left(s_{h}^{j}, a_{h}^{j}\right)^{\top}, G_{h, r}^{k}:=\sum_{j=1}^{k-1} \varphi\left(s_{h}^{j}, a_{h}^{j}\right) \varphi\left(s_{h}^{j}, a_{h}^{j}\right)^{\top}, g_{h, p}^{k}:=$ $\sum_{j=1}^{k-1} \phi_{h}^{k}\left(s_{h}^{j}, a_{h}^{j}\right) V_{h+1}^{j}\left(s_{h+1}^{j}\right)$ and $g_{h, r}^{k}:=\sum_{j=1}^{k-1} \varphi\left(s_{h}^{j}, a_{h}^{j}\right) r_{h}^{j}\left(s_{h}^{j}, a_{h}^{j}\right)$, all of which are in the form of sums. This motivates us to consider the binary counting mechanism (Chan et al., 2011), which is a tree-based utility-efficient scheme for releasing private counts. In the following, let's take the design of $Z_{h, p}^{k}$ and $z_{h, p}^{k}$ as an example and the same method applies to $Z_{h, r}^{k}$ and $z_{h, r}^{k}$. We first initialize $2 H$ counters, $\mathcal{B}_{h, p, 1}$ and $\mathcal{B}_{h, p, 2}$ for each $h \in[H]$. In particular, $\mathcal{B}_{h, p, 1}$ maintains a binary interval tree in which each node represents a partial sum (P-sum) in the form of $\Sigma_{p, 1}[i, j]=\sum_{k=i}^{j} \phi_{h}^{k}\left(s_{h}^{k}, a_{h}^{k}\right) \phi_{h}^{k}\left(s_{h}^{k}, a_{h}^{k}\right)^{\top}$ involving the features in episodes $i$ through $j$. Each leaf node represents a P-sum with a single episode (i.e., $i=j$ and hence the tree has $k-1$ leaf nodes at the start of episode $k$ ), and each interior node represents the range of episodes covered by its children. The same construction applies to $\mathcal{B}_{h, p, 2}$ with the P-sum given by $\Sigma_{p, 2}[i, j]=\sum_{k=i}^{j} \phi_{h}^{k}\left(s_{h}^{k}, a_{h}^{k}\right) V_{h+1}^{k}\left(s_{h+1}^{k}\right)$. Now, to release the private version of $G_{h, p}^{k}$ for each $k$ denoted by $\widehat{G}_{h, p}^{k}, \mathcal{B}_{h, p, 1}$ first release a noisy P-sum $\widehat{\Sigma}_{p, 1}[i, j]$ corresponding to each node in the tree. Here $\widehat{\Sigma}_{p, 1}[i, j]$ is obtained by perturbing both $(p, q)$-th and $(q, p)$-th, $1 \leq p \leq q \leq d_{1}$, entries of $\Sigma_{p, 1}[i, j]$ with i.i.d Gaussian noise $\mathcal{N}\left(0, \sigma_{p, 1}^{2}\right)^{3}$. Then the private $\widehat{G}_{h, p}^{k}$ is computed by summing up the noisy P-sums that uniquely cover the range $[1, k-1]$. Algorithmically speaking, $\mathcal{B}_{h, p, 1}$ is constructed and maintained by simply replacing the input stream of 0/1 bit in Algorithm 2 of (Chan et al., 2011) with the stream of $\left\{\phi_{h}^{k}\left(s_{h}^{k}, a_{h}^{k}\right) \phi_{h}^{k}\left(s_{h}^{k}, a_{h}^{k}\right)^{\top}\right\}_{k=1}^{K}$ in our case. To guarantee the resultant matrix is still positive definite, $\mathcal{B}_{h, p, 1}$ shifts $\widehat{G}_{h, p}^{k}$ by $\gamma I$ for some $\gamma>0$ specified later. Thus, the final matrix $\Lambda_{h, p}^{k}$ is the sum of non-private statistics $G_{h, p}^{k}$ and noise matrix $Z_{h, p}^{k}$, where $Z_{h, p}^{k}=N_{h, p}^{k}+\gamma I$ and $N_{h, p}^{k}$ is the total noise added via the summation of noisy P-sums. Similarly, for

\footnotetext{
${ }^{3}$ This will ensure symmetric of the P-sums even after adding noise.
} 
the counter $\mathcal{B}_{h, p, 2}$, the noisy P-sum $\widehat{\Sigma}_{p, 2}[i, j]$ is generated by perturbing each element of the $d_{1} \times 1$ vector with i.i.d Gaussian noise $\mathcal{N}\left(0, \sigma_{p, 2}^{2}\right)$. Then, the private $\widehat{g}_{h, p}^{k}$ is computed by summing up the noisy P-sums that uniquely cover the range $[1, k-1]$. As a result, the final vector $u_{h, p}^{k}$ is the sum of $g_{h, p}^{k}$ and $z_{h, p}^{k}$, where $z_{h, p}^{k}$ is the total noise added in the covering noisy P-sums. We follow the same way to maintain the counters $\mathcal{B}_{h, r, 1}$ and $\mathcal{B}_{h, r, 2}$ to privatize $G_{h, r}^{k}$ and $g_{h, r}^{k}$ and hence obtaining $Z_{h, r}^{k}$ and $z_{h, r}^{k}$. Note that, at the end of each episode, the counters only need to store noisy P-sums required for computing private statistics at future episodes, and hence can safely discard P-sums that are no longer needed.

One key problem in differential privacy is to balance between privacy guarantee and utility (i.e., regret in our case). That is, if more noise is added during the process, it becomes easier to guarantee privacy but meanwhile suffers from a worse regret. The distinguishing feature of binary counting mechanism discussed above is the ability to maintain a nice trade-off between privacy and utility, which is demonstrated via the following fundamental properties (Chan et al., 2011).

Lemma 6.1 (Fundamental properties of binary counting mechanism). Let $m:=\lceil\log K\rceil$. For all the counters $\mathcal{B}_{h, p, 1}, \mathcal{B}_{h, p, 2}, \mathcal{B}_{h, r, 1}$ and $\mathcal{B}_{h, r, 2}$ introduced above, we have

(i) Each episode appears only in at most $m$ P-sums.

(ii) There are at most $m P$-sums that uniquely cover the range $[1, k-1], \forall k \in[K]$.

In the following, we will rely on the two properties in Lemma 6.1 to analyze the privacy and regret guarantees, respectively. Before that, we first formally define two private RL algorithms by instantiating the REgularizer in Algorithm 1 and 2 with the PRIVATIZER that consists of the four counters $\mathcal{B}_{h, p, 1}, \mathcal{B}_{h, p, 2}$, $\mathcal{B}_{h, r, 1}$ and $\mathcal{B}_{h, r, 2}$ introduced above.

Definition 6.2 (Private-Linopt-VI). Private-Linopt-VI is defined as an instance of Algorithm 1 with the REGULARIZER being instantiated by a PRIVATIZER that consists of the four private counters $\mathcal{B}_{h, p, 1}, \mathcal{B}_{h, p, 2}, \mathcal{B}_{h, r, 1}$ and $\mathcal{B}_{h, r, 2}$.

Definition 6.3 (Private-Linopt-PO). Private-Linopt-PO is defined as an instance of Algorithm 2 with the REGULARIZER being instantiated by a PRIVATIZER that consists of the four private counters $\mathcal{B}_{h, p, 1}, \mathcal{B}_{h, p, 2}, \mathcal{B}_{h, r, 1}$ and $\mathcal{B}_{h, r, 2}$

\subsection{Privacy Guarantee}

In this section, we will show that both private algorithms provide JDP guarantee, which is summarized in the following theorem.

Theorem 6.4. Let Assumption 1 hold. For any $\varepsilon>0$ and $\delta \in(0,1]$, both Private-LinOpt-VI and Private-Linopt-POcan guarantee $O(\varepsilon, \delta)-J D P$.

Proof. We first show that with proper choices of noise parameter in the four counters (i.e., $\sigma_{p, 1}, \sigma_{p, 2}, \sigma_{r, 1}, \sigma_{r, 2}$ ), all the four counters are $(\varepsilon / 4, \delta / 4)$-DP. We begin with the counter $\mathcal{B}_{h, p, 1}$. To this end, we need to determine a global upper bound $\Delta_{p, 1}$ over the $L_{2}$-sensitivity so as to apply Gaussian mechanism (Dwork et al., 2014). Here, $\Delta_{p, 1}$ encodes the maximum change in the Frobenious norm of each P-sum if the state-action pair of a single episode is changed. By the boundedness assumptions in Assumption 1, we have $\left\|\phi_{h}^{k}(s, a)\right\| \leq$ $\sqrt{d_{1}} H, \forall s, a, h, k$, and hence $\Delta_{p, 1}=d_{1} H^{2} \leq d H^{2}$. Since the noisy P-sums are obtained via Gaussian noise, we have that each $\widehat{\Sigma}_{p, 1}[i, j]$ is $\left(\Delta_{p, 1}^{2} / 2 \sigma_{p, 1}^{2}\right)$-CDP (Bun and Steinke, 2016, Proposition 1.6). Now, by the 
property (i) in Lemma 6.1 and the composition property of DP, the whole counter $\mathcal{B}_{h, p, 1}$ is $\left(m \Delta_{p, 1}^{2} / 2 \sigma_{p, 1}^{2}\right)$ CDP, and thus, in turn, $\left(\frac{m \Delta_{p, 1}^{2}}{2 \sigma_{p, 1}^{2}}+2 \sqrt{\frac{m \Delta_{p, 1}^{2}}{2 \sigma_{p, 1}^{2}} \ln \left(\frac{4}{\delta}\right)}, \frac{\delta}{4}\right)$-DP for any $\delta>0$ (Bun and Steinke, 2016, Lemma 3.5). Now, setting $\sigma_{p, 1}^{2}=32 m \Delta_{p, 1}^{2} \ln (4 / \delta) / \varepsilon^{2}$, we can ensure that $\mathcal{B}_{h, p, 1}$ is $O(\varepsilon / 4, \delta / 4)$-DP. We then follow the same approach to establish that $\mathcal{B}_{h, p, 2}$ is $O(\varepsilon / 4, \delta / 4)$-DP with $\sigma_{p, 2}^{2}=32 m \Delta_{p, 2}^{2} \ln (4 / \delta) / \varepsilon^{2}$ and $\Delta_{p, 2}=\sqrt{d_{1}} H^{2} \leq \sqrt{d} H^{2}$. Similarly, we have $\mathcal{B}_{h, r, 1}$ and $\mathcal{B}_{h, r, 2}$ are both $O(\varepsilon / 4, \delta / 4)$-DP with $\sigma_{r, 1}^{2}=\sigma_{r, 2}^{2}=32 m \Delta^{2} \ln (4 / \delta) / \varepsilon^{2}$ with $\Delta=\Delta_{r, 1}=\Delta_{r, 2}=1$.

To prove the final result, we now use the billboard lemma (Hsu et al., 2016, Lemma 9), which states that an algorithm is JDP under continual observation if the output sent to each user is a function of the user's private data and a common quantity computed using standard differential privacy. Note that at each step $h$ of any episode $k$, in both algorithms, the estimators (e.g., $\widehat{\theta}_{h, p}^{k}$ ) and bonus terms (e.g., $\Gamma_{h, p}^{k}$ ) are computed via the four counters, each with $O(\varepsilon / 4, \delta / 4)$-DP guarantee. By composition and post-processing properties of DP, we can conclude that the sequences of policies $\left\{\pi^{k}\right\}_{k \in[K]}$ are computed using an $O(\varepsilon, \delta)$ DP mechanism. Now, the actions $\left\{a_{h}^{k}\right\}_{h \in H}$ during episode $k$ are generated via the policy $\pi^{k}$ and the user's data $s_{h}^{k}$ as $a_{h}^{k}=\pi^{k}\left(s_{h}^{k}\right)$. Then, by the billboard lemma, the composition of the controls $\left\{a_{h}^{k}\right\}_{k \in K, h \in H}$ sent to all the users is $O(H \varepsilon, H \delta)$-JDP. Hence, replacing $\varepsilon$ and $\delta$ by $\varepsilon / H$ and $\delta / H$, yields the final result.

\subsection{Regret Guarantee}

In this section, we will utilize the general regret bounds for LinOpt-VI-Reg and LinOpt-PO-Reg to derive the private regret bounds. In particular, thanks to the results in Theorems 4.1 and 5.1, we only need to determine the three constants $\lambda_{\max }, \lambda_{\min }$ and $\nu$ under the PRIVATIZER (equivalently the REGULARIZER in Algorithms 1 and 2) such that Assumption 2 holds. To this end, we will use concentration results for random Gaussian matrix (Lemma B.4) and chi-square random variable (Lemma B.5) to arrive at the following theorem.

Theorem 6.5. Let Assumption 1 hold. For any privacy parameters $\varepsilon>0$ and $\delta \in(0,1]$, and for any $\alpha \in(0,1]$, with probability at least $1-4 \alpha$, Private-LinOpt-VI enjoys the regret bound

$$
\mathcal{R}(T)=O\left(d \sqrt{T H^{3}} \log (T / \alpha)+\frac{\log (H / \delta)^{1 / 4}}{\varepsilon^{1 / 2}} d^{7 / 4} \sqrt{T H^{4}} \log T \log (T / \alpha)^{1 / 4}\right) .
$$

Similarly, for any $\alpha \in(0,1]$, with probability at least $1-4 \alpha$, Private-LinOpt-PO enjoys the regret bound

$$
\mathcal{R}(T)=O\left(d \sqrt{T H^{3}} \log (T / \alpha)+\sqrt{T H^{3} \log |\mathcal{A}|}+\frac{\log (H / \delta)^{1 / 4}}{\varepsilon^{1 / 2}} d^{7 / 4} \sqrt{T H^{4}} \log T \log (T / \alpha)^{1 / 4}\right) .
$$

Moreover, if $\sqrt{\log |\mathcal{A}|}=O\left(\log \frac{H}{\alpha}+d \log \left(1+K H^{2}\right)\right)$, then Private-LinOpt-PO has the same regret bound as given in (4).

Proof. As mentioned above, we only need to verify that the PRIVATIZER satisfies Assumption 2 with proper choices of $\lambda_{\max , p}, \lambda_{\max , r}, \lambda_{\min , p}, \lambda_{\min , r}$ and $\nu_{p}, \nu_{r}$. We will illustrate the idea behind $\lambda_{\max , p}, \lambda_{\min , p}$ and $\nu_{p}$ here and the same approach applies to the other three constants. By property (ii) in Lemma 6.1, we know that the total noise added into $\Lambda_{h, p}^{k}$ is a summation of $m$ symmetric random Gaussian matrix. That is, $N_{h, p}^{k}$ is a symmetric matrix with its $(i, j)$-th entry, $1 \leq i \leq j \leq d_{1}$, being i.i.d $\mathcal{N}\left(0, m \sigma_{p, 1}^{2}\right)$. Therefore, by a 
tighter concentration bound for symmetric random Gaussian matrix (Lemma B.4 in the appendix), we have with probability at least $1-\alpha /(4 K H)$, for all $k \in[K], h \in[H]$

$$
\left\|N_{h, p}^{k}\right\| \leq \Sigma_{p, 1}:=\sigma_{p, 1} \sqrt{m}(4 \sqrt{d}+\sqrt{8 \log (8 K H / \alpha)}) .
$$

Recall that $Z_{h, p}^{k}$ is a shift version of $N_{h, p}^{k}$ to guarantee that it is positive definite. In particular, we choose $Z_{h, p}^{k}=N_{h, p}^{k}+2 \Sigma_{p, 1} I$, which leads to $\lambda_{\max , p}=3 \Sigma_{p, 1}$ and $\lambda_{\min , p}=\Sigma_{p, 1}$.

Similarly, the total noise in $u_{h, p}^{k}$ is a summation of $m$ random Gaussian vectors. That is, $z_{h, p}^{k}$ is a $d_{1} \times 1$ vector whose each entry is i.i.d $\mathcal{N}\left(0, m \sigma_{p, 2}^{2}\right)$. As a result, $\left\|z_{h, p}^{k}\right\|^{2} /\left(m \sigma_{p, 2}^{2}\right)$ a $\chi^{2}$-statistic with $d_{1}$ degrees of freedom, and hence by concentration bound of chi-square random variable (Lemma B.5 in the appendix), we have with probability at least $1-\alpha /(4 K H)$, for all $k \in[K], h \in[H]$

$$
\left\|z_{h, p}^{k}\right\| \leq \sqrt{m} \sigma_{p, 2}\left(\sqrt{d_{1}}+\sqrt{2 \log (4 K H / \alpha)}\right) .
$$

Thus, via a union bound, we have with probability at least $1-\alpha / 2$, for all $h \in[H]$ and $k \in[K]$,

$$
\left\|Z_{h, p}^{k}\right\| \leqslant \lambda_{\max , p}, \quad\left\|\left(Z_{h, p}^{k}\right)^{-1}\right\| \leqslant 1 / \lambda_{\min , p} \text { and }\left\|z_{h, p}^{k}\right\|_{\left(Z_{h, p}^{k}\right)^{-1}} \leqslant \nu_{p},
$$

where $\nu_{p}=\sigma_{p, 2} \sqrt{m / \Sigma_{p, 1}}\left(\sqrt{d_{1}}+\sqrt{2 \log (4 K H / \alpha)}\right)$. Moreover, via the same analysis, we also have with probability at least $1-\alpha / 2$, for all $h \in[H]$ and $k \in[K]$,

$$
\left\|Z_{h, r}^{k}\right\| \leqslant \lambda_{\max , r}, \quad\left\|\left(Z_{h, r}^{k}\right)^{-1}\right\| \leqslant 1 / \lambda_{\min , r} \text { and }\left\|z_{h, r}^{k}\right\|_{\left(Z_{h, r}^{k}\right)^{-1}} \leqslant \nu_{r}
$$

where $\lambda_{\max , r}=3 \lambda_{\min , r}=3 \Sigma_{r}, \nu_{p}=\sigma_{r, 2} \sqrt{m / \Sigma_{r}}\left(\sqrt{d_{2}}+\sqrt{2 \log (4 K H / \alpha)}\right)$ and

$$
\Sigma_{r}=\sigma_{r, 1} \sqrt{m}\left(4 \sqrt{d_{2}}+\sqrt{8 \log (8 K H / \alpha)}\right) .
$$

Recall that to guarantee $O(\varepsilon, \delta)$-JDP in Theorem 6.4, we choose $\sigma_{p, 1}=\frac{H \Delta_{p, 1}}{\varepsilon} \sqrt{32 m \ln (4 H / \delta)}, \sigma_{p, 2}=$ $\frac{H \Delta_{p, 2}}{\varepsilon} \sqrt{32 m \ln (4 H / \delta)}, \sigma_{r, 1}=\frac{H \Delta_{r, 1}}{\varepsilon} \sqrt{32 m \ln (4 H / \delta)}, \sigma_{r, 2}=\frac{H \Delta_{r, 2}}{\varepsilon} \sqrt{32 m \ln (4 H / \delta)}$ with $\Delta_{p, 1}=d_{1} H^{2} \leq$ $d H^{2}, \Delta_{p, 2}=\sqrt{d_{1}} H^{2} \leq \sqrt{d} H^{2}, \Delta_{r, 1}=\Delta_{r, 2}=1$. Substituting these constants into the regret bound in Theorems 4.1 and 5.1, yields the final results.

Remark 6.6 (Bound on $d$ ). Note that $d^{7 / 4}$ in the second term (i.e., private term) of both regret bounds comes from simply bounding both $d_{1}$ and $d_{2}$ by $d$. A tighter bound on d is straightforward if it is written in an explicit form of $d_{1}$ and $d_{2}$. Note that if we assume that $\|\phi(s, a)\| \leq H$ (as in (He et al., 2021b; Zhou et al., 2021a) ) rather than $\sqrt{d} H$ in Assumption 1, the private dependency on $d$ then reduces from $d^{7 / 4}$ to $d^{5 / 4}$.

Remark 6.7 (Comparisons with non-private algorithms). The non-private value-based algorithm UCRLVTR (Jia et al., 2020) enjoys a regret upper bound $\widetilde{O}\left(d \sqrt{H^{3} T}\right)$ and the non-private policy-based algorithm OPPO (Cai et al., 2020) has a regret upper bound $\widetilde{O}\left(d \sqrt{T H^{3}}+\sqrt{T H^{3} \log |\mathcal{A}|}\right)$. On the other hand, Private-LinOpt-VI has a regret bound $\widetilde{O}\left(d^{7 / 4} \sqrt{T H^{4}}\left(\log (H / \delta)^{1 / 4}\right) \sqrt{1 / \varepsilon}\right)$ and Private-LinOpt-PO has a regret bound $\widetilde{O}\left(d^{7 / 4} \sqrt{T H^{4}}\left(\log (H / \delta)^{1 / 4}\right) \sqrt{1 / \varepsilon}+\sqrt{T H^{3} \log |\mathcal{A}|}\right)$. 
Remark 6.8 (Comparisons tabular private RL result). We compare our results to (Vietri et al., 2020), which establishes regret for tabular value-based RL under $(\varepsilon, 0)-J D P$ guarantee by injecting Laplace noise via binary counting mechanism. In particular, the claimed regret is $\widetilde{O}\left(\sqrt{S A H^{3} T}+S^{2} A H^{3} / \varepsilon\right)$, i.e., both non-private and private terms are polynomial in terms of the number of states and actions while ours are independent of them. Moreover, the dependence of $\varepsilon$ is an additive term of $1 / \varepsilon$ in (Vietri et al., 2020) while ours have a multiplicative factor $1 / \sqrt{\varepsilon}$ under $(\varepsilon, \delta)$-JDP. Finally, since tabular MDP is a special case of linear mixture MDP, our established regret bounds directly imply bounds for the tabular case (although may not be optimal in general through this reduction.)

Remark 6.9 (General private RL design). As mentioned before, our two proposed algorithms ( LinOpt-VI-Reg and LinOpt-PO-Reg) along with their regret bounds (Theorems 4.1 and 5.1) provide a unified procedure to designing various private RL algorithms by varying the choice of REGULARIZER (or equivalently PRIVATIZER). This not only enables to design new private RL algorithms with JDP guarantees, but allows us to establish RL algorithms with LDP guarantees. In this case, instead of a central PRIVATIZER at the agent, a local PRIVATIZER at each user's side is used to inject independent Gaussian noise to user's trajectories before being collected by the agent. However, our analysis smoothly carries out and the only change is to determine the three new constants $\lambda_{\max }, \lambda_{\min }, \nu$ under stronger noise in the LDP setting, which can be obtained via the same approach in our current analysis.

\subsection{Discussions}

In this paper, for the purpose of theoretical analysis, we assume realizability, i.e. the MDP in fact lies within the class of linear mixture MDPs. One important future direction is to consider misspecified linear models (cf. (Jin et al., 2020; Lattimore et al., 2020)). Another important direction is to apply our flexible framework to MDPs with other types of function approximation, e.g., kernelized MDPs or overparameterized neural network (Yang et al., 2020) and generic MDPs (Ayoub et al., 2020). Specifically, at least in the kernelized MDPs settings (i.e., reproducing kernel Hilbert space (RKHS)), it turns out that our current analysis can be easily carried out by using the standard Quadrature Fourier Features (QFF) approximations (Mutnỳ and Krause, 2019). It is worth noting that one drawback of QFF approximation is that it only works with standard squared exponential kernel but not with more practical Matern kernel (Calandriello et al., 2019). One interesting question is whether it is possible to use adaptive approximation methods (e.g., Nyström Embedding (Calandriello et al., 2019)) that works for both kernels in the private RL learning. The difficulty here is that the truncated dimension is changing in each round. In the setting with general function approximations, it is still not clear how to generalize our current analysis with a computationally efficient private scheme such as the binary counting mechanism used in our paper. Finally, it will also be interesting to consider other privacy guarantees beyond JDP and LDP, e.g., shuffle model of $D P$ (Cheu et al., 2019), that achieves a smooth transition between JDP and LDP. This is particularly useful even in the simpler linear bandit setting as the regret under LDP is $O\left(T^{3 / 4}\right)$ (Zheng et al., 2020) while the regret under JDP is $O(\sqrt{T})$ (Shariff and Sheffet, 2018). Thus, one interesting question is whether the shuffle model of DP can be utilized to achieve a similar regret as in JDP while providing the same strong privacy guarantee as in LDP, which is one of ongoing works.

\section{$7 \quad$ Proofs of Theorems 4.1 and 5.1}

In this section, we present the detailed proofs for Theorems 4.1 and 5.1. To start with, we define the following filtration which will be frequently used in the proofs. This filtration can be regarded as the bandit- 
variant of the one in (Cai et al., 2020).

Definition 7.1 (Filtration). For any $(k, h) \in[K] \times[H]$, we define $\mathcal{F}_{k, h, 1}$ as the $\sigma$-algebra generated by the following state-action-reward sequence,

$$
\left\{\left(s_{i}^{\tau}, a_{i}^{\tau}, r_{i}^{\tau}\right)\right\}_{(\tau, i) \in[k-1] \times[H]} \cup\left\{\left(s_{i}^{k}, a_{i}^{k}, r_{i}^{k}\right)_{i \in[h]}\right\}
$$

and $\mathcal{F}_{k, h, 2}$ as the $\sigma$-algebra generated by

$$
\left\{\left(s_{i}^{\tau}, a_{i}^{\tau}, r_{i}^{\tau}\right)\right\}_{(\tau, i) \in[k-1] \times[H]} \cup\left\{\left(s_{i}^{k}, a_{i}^{k}, r_{i}^{k}\right)_{i \in[h]}\right\} \cup\left\{s_{h+1}^{k}\right\},
$$

where we define $s_{H+1}^{k}$ as a null state for any $k \in[K]$. The $\sigma$-algebra sequence $\left\{\mathcal{F}_{k, h, m}\right\}_{(k, h, m) \in[K] \times[H] \times 2}$ is a filtration with respect to the timestep index

$$
t(k, h, m)=2 H(k-1)+2(h-1)+m .
$$

In other words, for any $t(k, h, m) \leq t\left(k^{\prime}, h^{\prime}, m^{\prime}\right)$, it holds that $\mathcal{F}_{k, h, m} \subseteq \mathcal{F}_{k^{\prime}, h^{\prime}, m^{\prime}}$.

By the definition, we immediately see that the estimated value function $V_{h}^{k}$ and $Q$-function $Q_{h}^{k}$ in both algorithms are measurable to $\mathcal{F}_{k, 1,1}$ as they are obtained based on the $(k-1)$ historical trajectories. Moreover, throughout the proofs, we let $\mathcal{E}(\alpha):=\left\{\theta_{h, p} \in \mathcal{C}_{h, p}^{k}\right.$ and $\left.\theta_{h, r} \in \mathcal{C}_{h, r}^{k}, \forall h \in[H], k \in[K]\right\}$ denote the 'good' event, which by Lemma 3.1, holds with probability at least $1-3 \alpha$.

\subsection{Proof of Theorem 4.1}

We first show that the estimated $Q$-function and value function in Algorithm 1 is optimistic. First note that, under the nice event given by $\mathcal{E}(\alpha)$, we have that for all $s, a, k, h$

$$
\varphi(s, a)^{\top} \hat{\theta}_{h, r}^{k}+\phi_{h}^{k}(s, a)^{\top} \widehat{\theta}_{h, p}^{k}+\left(\Gamma_{h, p}^{k}+\Gamma_{h, r}^{k}\right)(s, a) \geq r_{h}(s, a)+\left[\mathbb{P}_{h} V_{h+1}^{k}\right](s, a) \geq 0
$$

Thus, under event $\mathcal{E}(\alpha)$, we can remove the truncation at zero in the update of $Q$ function in Algorithm 1 .

Lemma 7.2. Under event $\mathcal{E}(\alpha)$, for any $s, a, k, h$, we have $Q_{h}^{k}(s, a) \geq Q_{h}^{*}(s, a)$ and $V_{h}^{k}(s, a) \geq V_{h}^{*}(s, a)$.

Proof. The proof is standard (cf. (Ding et al., 2021, Lemma C.4)). We present the proof here for ease of reference. The result is proved by induction. First note that the base case for $h=H+1$ holds trivially since all the values are zero. Fix a $k$, suppose the result holds $h+1$, i.e., $Q_{h}^{k+1}(\cdot, \cdot) \geq Q_{h+1}^{*}(\cdot, \cdot), V_{h+1}^{k}(\cdot, \cdot) \geq$ $V_{h+1}^{*}(\cdot, \cdot)$. Given $s, a$, if $Q_{h}^{k}(s, a) \geq H$, then $Q_{h}^{k}(s, a) \geq H \geq Q_{h}^{*}(s, a)$. Otherwise, we have

$$
\begin{aligned}
& Q_{h}^{k}(s, a)-Q_{h}^{*}(s, a) \\
\stackrel{(a)}{=} & \left\langle\widehat{\theta}_{h, p}^{k}-\theta_{h, p}, \phi_{h}^{k}\left(s_{h}^{k}, a_{h}^{k}\right)\right\rangle+\left\langle\widehat{\theta}_{h, r}^{k}-\theta_{h, r}, \varphi\left(s_{h}^{k}, a_{h}^{k}\right)\right\rangle+\left[\mathbb{P}_{h} V_{h+1}^{k}\right]\left(s_{h}^{k}, a_{h}^{k}\right) \\
& +\beta_{p}(\alpha)\left\|\phi_{h}^{k}\left(s_{h}^{k}, a_{h}^{k}\right)\right\|_{\left(\Lambda_{h, p}^{k}\right)^{-1}}+\beta_{r}(\alpha)\left\|\varphi\left(s_{h}^{k}, a_{h}^{k}\right)\right\|_{\left(\Lambda_{h, r}^{k}\right)^{-1}}-\left[\mathbb{P}_{h} V_{h+1}^{*}\right]\left(s_{h}^{k}, a_{h}^{k}\right) \\
& \stackrel{(b)}{\geq}\left[\mathbb{P}_{h} V_{h+1}^{k}\right](s, a)-\left[\mathbb{P}_{h} V_{h+1}^{*}\right](s, a) \\
& \stackrel{(c)}{\geq} 0
\end{aligned}
$$

where (a) holds by adding and subtracting the same term, which holds by the linear kernel MDP model; (b) holds by event $\mathcal{E}(\alpha)$ and Cauchy-Schwarz inequality; (c) holds by the induction assumption and the fact that $\mathbb{P}_{h}$ is a monotone operator with respect the partial ordering of functions. Thus, for all $(s, a)$, we have established that $Q_{h}^{k}(s, a) \geq Q_{h}^{*}(s, a)$, which directly implies that $V_{h}^{k}(s, a) \geq V_{h}^{*}(s, a)$. 
Now, we are ready to present the proof of Theorem 4.1.

Proof. First, we have under event $\mathcal{E}(\alpha)$,

$$
\begin{aligned}
& V_{h}^{k}\left(s_{h}^{k}\right)-V_{h}^{\pi^{k}}\left(s_{h}^{k}\right) \\
\stackrel{(a)}{\leq} & \left\langle\widehat{\theta}_{h, p}^{k}, \phi_{h}^{k}\left(s_{h}^{k}, a_{h}^{k}\right)\right\rangle+\left\langle\widehat{\theta}_{h, r}^{k}, \varphi\left(s_{h}^{k}, a_{h}^{k}\right)\right\rangle+\beta_{p}(\alpha)\left\|\phi_{h}^{k}\left(s_{h}^{k}, a_{h}^{k}\right)\right\|_{\left(\Lambda_{h, p}^{k}\right)^{-1}}+\beta_{r}(\alpha)\left\|\varphi\left(s_{h}^{k}, a_{h}^{k}\right)\right\|_{\left(\Lambda_{h, r}^{k}\right)^{-1}} \\
& r_{h}\left(s_{h}^{k}, a_{h}^{k}\right)-\left[\mathbb{P}_{h} V_{h+1}^{\pi^{k}}\right]\left(s_{h}^{k}, a_{h}^{k}\right) \\
\stackrel{(b)}{=} & \left\langle\widehat{\theta}_{h, p}^{k}-\theta_{h, p}, \phi_{h}^{k}\left(s_{h}^{k}, a_{h}^{k}\right)\right\rangle+\left\langle\widehat{\theta}_{h, r}^{k}-\theta_{h, r}, \varphi\left(s_{h}^{k}, a_{h}^{k}\right)\right\rangle+\left[\mathbb{P}_{h} V_{h+1}^{k}\right]\left(s_{h}^{k}, a_{h}^{k}\right) \\
& +\beta_{p}(\alpha)\left\|\phi_{h}^{k}\left(s_{h}^{k}, a_{h}^{k}\right)\right\|_{\left(\Lambda_{h, p}^{k}\right)^{-1}}+\beta_{r}(\alpha)\left\|\varphi\left(s_{h}^{k}, a_{h}^{k}\right)\right\|_{\left(\Lambda_{h, r}^{k}\right)^{-1}}-\left[\mathbb{P}_{h} V_{h+1}^{\pi^{k}}\right]\left(s_{h}^{k}, a_{h}^{k}\right) \\
\stackrel{(c)}{\leq} & {\left[\mathbb{P}_{h} V_{h+1}^{k}\right]\left(s_{h}^{k}, a_{h}^{k}\right)-\left[\mathbb{P}_{h} V_{h+1}^{\pi^{k}}\right]\left(s_{h}^{k}, a_{h}^{k}\right)+2 \beta_{p}(\alpha)\left\|\phi_{h}^{k}\left(s_{h}^{k}, a_{h}^{k}\right)\right\|_{\left(\Lambda_{h, p}^{k}\right)^{-1}}+2 \beta_{r}(\alpha)\left\|\varphi\left(s_{h}^{k}, a_{h}^{k}\right)\right\|_{\left(\Lambda_{h, r}^{k}\right)^{-1}} }
\end{aligned}
$$

where (a) holds by the update of $V_{h}^{k}$ in Algorithm 1, the Bellman equation for $V_{h}^{\pi^{k}}$, and the greedy selection of the algorithm; (b) holds by the linear mixture MDP model; (c) holds by Cauchy-Schwarz inequality and the event of $\mathcal{E}(\alpha)$.

Now, by using Lemma 7.2, we can further bound (6) as follows.

$$
\begin{aligned}
& V_{h}^{k}\left(s_{h}^{k}\right)-V_{h}^{\pi^{k}}\left(s_{h}^{k}\right) \\
& \stackrel{(a)}{\leq} \min \left\{H,\left[\mathbb{P}_{h} V_{h+1}^{k}\right]\left(s_{h}^{k}, a_{h}^{k}\right)-\left[\mathbb{P}_{h} V_{h+1}^{\pi^{k}}\right]\left(s_{h}^{k}, a_{h}^{k}\right)+2 \beta_{p}(\alpha)\left\|\phi_{h}^{k}\left(s_{h}^{k}, a_{h}^{k}\right)\right\|_{\left(\Lambda_{h, p}^{k}\right)^{-1}}+2 \beta_{r}(\alpha)\left\|\varphi\left(s_{h}^{k}, a_{h}^{k}\right)\right\|_{\left(\Lambda_{h, r}^{k}\right)^{-1}}\right\} \\
& \stackrel{(b)}{\leq} \min \left\{H, 2 \beta_{p}(\alpha)\left\|\phi_{h}^{k}\left(s_{h}^{k}, a_{h}^{k}\right)\right\|_{\left(\Lambda_{h, p}^{k}\right)^{-1}}+2 \beta_{r}(\alpha)\left\|\varphi\left(s_{h}^{k}, a_{h}^{k}\right)\right\|_{\left(\Lambda_{h, r}^{k}\right)^{-1}}\right\}+\left[\mathbb{P}_{h} V_{h+1}^{k}\right]\left(s_{h}^{k}, a_{h}^{k}\right)-\left[\mathbb{P}_{h} V_{h+1}^{\pi^{k}}\right]\left(s_{h}^{k}, a_{h}^{k}\right) \\
& \stackrel{(c)}{\leq} 2 \beta_{p}(\alpha) \min \left\{1,\left\|\phi_{h}^{k}\left(s_{h}^{k}, a_{h}^{k}\right)\right\|_{\left(\Lambda_{h, p}^{k}\right)^{-1}}\right\}+2 H \beta_{r}(\alpha) \min \left\{1,\left\|\varphi\left(s_{h}^{k}, a_{h}^{k}\right)\right\|_{\left(\Lambda_{h, r}^{k}\right)^{-1}}\right\} \\
& \quad+\left[\mathbb{P}_{h} V_{h+1}^{k}\right]\left(s_{h}^{k}, a_{h}^{k}\right)-\left[\mathbb{P}_{h} V_{h+1}^{\pi^{k}}\right]\left(s_{h}^{k}, a_{h}^{k}\right)
\end{aligned}
$$

where (a) holds by the fact that value function is bounded in the range $[0, H]$; (b) is true since $V_{h+1}^{k}(\cdot) \geq$ $V_{h+1}^{\pi^{k}}(\cdot)$ by Lemma 7.2 on event $\mathcal{E}(\alpha)$; (c) is truce since $2 \beta_{p}(\alpha) \geq H$ and $2 H \beta_{r}(\alpha) \geq H$ by the definitions of $\beta_{p}(\alpha)$ and $\beta_{r}(\alpha)$ in Lemma 3.1. Therefore, we have

$$
\begin{aligned}
& V_{h}^{k}\left(s_{h}^{k}\right)-V_{h}^{\pi^{k}}\left(s_{h}^{k}\right)-\left(V_{h+1}^{k}\left(s_{h+1}^{k}\right)-V_{h+1}^{\pi^{k}}\left(s_{h+1}^{k}\right)\right) \\
\leq & 2 \beta_{p}(\alpha) \min \left\{1,\left\|\phi_{h}^{k}\left(s_{h}^{k}, a_{h}^{k}\right)\right\|_{\left(\Lambda_{h, p}^{k}\right)^{-1}}\right\}+2 H \beta_{r}(\alpha) \min \left\{1,\left\|\varphi\left(s_{h}^{k}, a_{h}^{k}\right)\right\|_{\left(\Lambda_{h, r}^{k}\right)^{-1}}\right\} \\
& +\mathbb{P}_{h}\left[V_{h+1}^{k}-V_{h}^{\pi^{k}}\right]\left(s_{h}^{k}, a_{h}^{k}\right)-\left[V_{h+1}^{k}-V_{h}^{\pi^{k}}\right]\left(s_{h+1}^{k}\right) .
\end{aligned}
$$


Therefore, taking a summation over $k \in[K]$ and $h \in[H]$, yields that under event $\mathcal{E}(\alpha)$

$$
\begin{aligned}
\sum_{k=1}^{K} V_{1}^{k}\left(s_{1}^{k}\right)-V_{1}^{\pi^{k}}\left(s_{1}^{k}\right) & \leq \underbrace{\sum_{k=1}^{K} \sum_{h=1}^{H} 2 \beta_{p}(\alpha) \min \left\{1,\left\|\phi_{h}^{k}\left(s_{h}^{k}, a_{h}^{k}\right)\right\|_{\left.\left(\Lambda_{h, p}^{k}\right)^{-1}\right\}}\right\}}_{\mathcal{T}_{1}} \\
& +\underbrace{\sum_{k=1}^{K} \sum_{h=1}^{H} 2 H \beta_{r}(\alpha) \min \left\{1,\left\|\varphi\left(s_{h}^{k}, a_{h}^{k}\right)\right\|_{\left(\Lambda_{h, r}^{k}\right)^{-1}}\right\}}_{\mathcal{T}_{2}} \\
& +\underbrace{\sum_{k=1}^{K} \sum_{h=1}^{H} \mathbb{P}_{h}\left[V_{h+1}^{k}-V_{h}^{\pi^{k}}\right]\left(s_{h}^{k}, a_{h}^{k}\right)-\left[V_{h+1}^{k}-V_{h}^{\pi^{k}}\right]\left(s_{h+1}^{k}\right)}_{\mathcal{T}_{3}} .
\end{aligned}
$$

Now, we are left to bound each of the two terms. First, $\mathcal{T}_{3}$ is a summation of a bounded martingale difference sequence, with boundedness being $H$. To see this, we let $Y_{k, h}:=\mathbb{P}_{h}\left[V_{h+1}^{k}-V_{h}^{\pi^{k}}\right]\left(s_{h}^{k}, a_{h}^{k}\right)-\left[V_{h+1}^{k}-\right.$ $\left.V_{h}^{\pi^{k}}\right]\left(s_{h+1}^{k}\right)$. Then, we have $Y_{k, h} \in \mathcal{F}_{k, h, 2}$ and $\mathbb{E}\left[Y_{k, h} \mid \mathcal{F}_{k, h, 1}\right]=0$. Thus, by the Azuma-Hoeffding inequality, we have that for any fixed $\alpha \in(0,1]$, with probability at least $1-\alpha$,

$$
\mathcal{T}_{3} \leq H \sqrt{2 K H \log (1 / \alpha)}
$$

To bound $\mathcal{T}_{1}$ and $\mathcal{T}_{2}$, we will use the standard elliptical potential lemma (cf. (Abbasi-Yadkori et al., 2011, Lemma 11)). In particular, we have

$$
\begin{aligned}
& \mathcal{T}_{1} \stackrel{(a)}{\leq} 2 \beta_{p}(\alpha) \sqrt{K H} \sqrt{\sum_{k=1}^{K} \sum_{h=1}^{H} \min \left\{1,\left\|\phi_{h}^{k}\left(s_{h}^{k}, a_{h}^{k}\right)\right\|_{\left(\Lambda_{h, p}^{k}\right)^{-1}}^{2}\right\}} \\
& \quad \stackrel{(b)}{\leq} 2 \beta_{p}(\alpha) \sqrt{K H} \sqrt{2 H d_{1} \log \left(1+K H^{2} /\left(\lambda_{\min , p}\right)\right)},
\end{aligned}
$$

where (a) holds by Cauchy-Schwarz inequality; (b) follows from Lemma 11 in (Abbasi-Yadkori et al., 2011) and the last step in the proof of Lemma 3.1. Similarly, we have

$$
\begin{aligned}
\mathcal{T}_{2} & \leq 2 H \beta_{r}(\alpha) \sqrt{K H} \sqrt{\sum_{k=1}^{K} \sum_{h=1}^{H} \min \left\{1,\left\|\varphi\left(s_{h}^{k}, a_{h}^{k}\right)\right\|_{\left(\Lambda_{h, r}^{k}\right)^{-1}}^{2}\right\}} \\
& \leq 2 H \beta_{r}(\alpha) \sqrt{K H} \sqrt{2 H d_{2} \log \left(1+K /\left(d_{2} \lambda_{\min , r}\right)\right)}
\end{aligned}
$$

Finally, note that $V_{1}^{*}\left(s_{1}^{k}\right) \leq V_{1}^{k}\left(s_{1}^{k}\right)$ for all $k$ by Lemma 7.2. Putting everything together, we have for any $\alpha \in(0,1]$, with probability at least $1-4 \alpha$, Algorithm 1 achieves a regret bound

$$
\mathcal{R}(T)=O\left(\sqrt{T H^{3}}\left(\log \frac{H}{\alpha}+d \kappa_{\lambda_{\min }}\right)+\sqrt{T H d \kappa_{\lambda_{\min }}}\left(\sqrt{d \lambda_{\max }}+\nu\right)\right)
$$

where $\kappa_{\lambda_{\min }}:=\log \left(1+K H^{2} /\left(\lambda_{\min }\right)\right)$ and $\lambda_{\min }=\min \left\{\lambda_{\min , p}, \lambda_{\min , r}\right\}, \lambda_{\max }=\max \left\{\lambda_{\max , p}, \lambda_{\max , r}\right\}$ and $\nu=\max \left\{\nu_{p}, \nu_{r}\right\}$ 


\section{Proof of Theorem 5.1}

Proof. We start with the following standard regret decomposition (cf. (Cai et al., 2020, Lemma 4.2)).

$$
\begin{aligned}
\mathcal{R}(T) & =\sum_{k=1}^{K}\left(V_{1}^{*}\left(s_{1}^{k}\right)-V_{1}^{\pi_{k}}\left(s_{1}^{k}\right)\right) \\
& =\underbrace{\sum_{k=1}^{K} \sum_{h=1}^{H} \mathbb{E}_{\pi^{*}}\left[\left\langle Q_{h}^{k}\left(s_{h}^{k}\right), \pi_{h}^{*}\left(\cdot \mid s_{h}\right)-\pi_{h}^{\pi_{k}}\left(\cdot \mid s_{h}\right)\right\rangle \mid s_{1}=s_{1}^{k}\right]}_{(i)}+\underbrace{\mathcal{M}_{K, H, 2}}_{(i i)} \\
& +\underbrace{\sum_{k=1}^{K} \sum_{h=1}^{H}\left(\mathbb{E}_{\pi^{*}}\left[\iota_{h}^{k}\left(x_{h}, a_{h}\right) \mid s_{1}=s_{1}^{k}\right]-\iota_{h}^{k}\left(s_{h}^{k}, a_{h}^{k}\right)\right)}_{(i i i)},
\end{aligned}
$$

where $\iota_{h}^{k}:=r_{h}+\mathbb{P}_{h} V_{h+1}^{k}-Q_{h}^{k}$ and $\mathbb{E}_{\pi}[\cdot]$ is taking expectation with respect to $\pi$ with transitions $\mathbb{P}_{h}$.

Here, $\left\{\mathcal{M}_{k, h, m}\right\}_{(k, h, m) \in[K] \times[H] \times[2]}$ is a martingale adapted to the filtration $\left\{\mathcal{F}_{k, h,}\right\}_{(k, h, m) \in[K] \times[H] \times[2]}$ with respect to the timestep in Definition 7.1. Specifically, we have

$$
\mathcal{M}_{K, H, 2}=\sum_{k=1}^{K} \sum_{h=1}^{H}\left(D_{k, h, 1}+D_{k, h, 2}\right),
$$

where

$$
\begin{aligned}
& D_{k, h, 1}=\left(\mathbb{J}_{k, h}\left(Q_{h}^{k}-Q_{h}^{\pi^{k}}\right)\right)\left(s_{h}^{k}\right)-\left(Q_{h}^{k}-Q_{h}^{\pi^{k}}\right)\left(s_{h}^{k}, a_{h}^{k}\right) \\
& D_{k, h, 2}=\mathbb{P}_{h}\left[V_{h+1}^{k}-V_{h}^{\pi^{k}}\right]\left(s_{h}^{k}, a_{h}^{k}\right)-\left[V_{h+1}^{k}-V_{h}^{\pi^{k}}\right]\left(s_{h+1}^{k}\right),
\end{aligned}
$$

and $\left(\mathbb{J}_{k, h} f\right)(x):=\left\langle f(x, \cdot), \pi_{h}^{k}(\cdot \mid x)\right\rangle$. Thus, by the boundedness of value functions and $Q$-functions along with Azuma-Hoeffding inequality, we have $(i i) \leq 4 H \sqrt{2 T \log (1 / \alpha)}$. The first term can be bounded by using standard property of mirror-descent update and the fact that $\pi^{1}$ is a uniform distribution. In particular, as in (Cai et al., 2020, Eq. C.2), we have $(i) \leq \sqrt{2 H^{3} T \log |\mathcal{A}|}$ when $\eta=\sqrt{2 \log |\mathcal{A}| /(H T)}$ in Algorithm 2. The key part is to bound the third term. To this end, we will show that under good event $\mathcal{E}(\alpha)$, we have for all $s, a, k, h$,

$$
2\left(\Gamma_{h, p}^{k}+\Gamma_{h, r}^{k}\right)(s, a) \geq-\iota_{h}^{k}(s, a) \geq 0 .
$$

To show this result, we first note that under $\mathcal{E}(\alpha)$, for all $s, a, k, h$

$$
\varphi(s, a)^{\top} \widehat{\theta}_{h, r}^{k}+\phi_{h}^{k}(s, a)^{\top} \widehat{\theta}_{h, p}^{k}+\left(\Gamma_{h, p}^{k}+\Gamma_{h, r}^{k}\right)(s, a) \geq r_{h}(s, a)+\left[\mathbb{P}_{h} V_{h+1}^{k}\right](s, a) \geq 0
$$

Thus, under event $\mathcal{E}(\alpha)$, we can remove the truncation at zero in the update of $Q$ function in Algorithm 2. Thus, we have

$$
\begin{aligned}
-\iota_{h}^{k}(s, a) & =Q_{h}^{k}(s, a)-\left(r_{h}(s, a)+\left[\mathbb{P}_{h} V_{h+1}^{k}\right](s, a)\right) \\
& \leq \varphi(s, a)^{\top} \widehat{\theta}_{h, r}^{k}+\phi_{h}^{k}(s, a)^{\top} \widehat{\theta}_{h, p}^{k}+\left(\Gamma_{h, p}^{k}+\Gamma_{h, r}^{k}\right)(s, a)-\left(r_{h}(s, a)+\left[\mathbb{P}_{h} V_{h+1}^{k}\right](s, a)\right) \\
& \leq 2\left(\Gamma_{h, p}^{k}+\Gamma_{h, r}^{k}\right)(s, a) .
\end{aligned}
$$


Meanwhile, we have under event $\mathcal{E}(\alpha)$

$$
\begin{aligned}
& \iota_{h}^{k}(s, a) \\
= & \left(r_{h}(s, a)+\left[\mathbb{P}_{h} V_{h+1}^{k}\right](s, a)\right)-Q_{h}^{k}(s, a) \\
= & \left(r_{h}(s, a)+\left[\mathbb{P}_{h} V_{h+1}^{k}\right](s, a)\right)-\min \left\{H, \varphi(s, a)^{\top} \widehat{\theta}_{h, r}^{k}+\phi_{h}^{k}(s, a)^{\top} \widehat{\theta}_{h, p}^{k}+\left(\Gamma_{h, p}^{k}+\Gamma_{h, r}^{k}\right)(s, a)\right\} \\
= & \max \left\{r_{h}(s, a)+\left[\mathbb{P}_{h} V_{h+1}^{k}\right](s, a)-H, r_{h}(s, a)+\left[\mathbb{P}_{h} V_{h+1}^{k}\right](s, a)-\varphi(s, a)^{\top} \widehat{\theta}_{h, r}^{k}-\phi_{h}^{k}(s, a)^{\top} \widehat{\theta}_{h, p}^{k}-\left(\Gamma_{h, p}^{k}+\Gamma_{h, r}^{k}\right)(s, a)\right\} \\
\leq & 0
\end{aligned}
$$

Therefore, similar to the proof of Theorem 4.1, we have

$$
\text { (iii) }=O\left(\sqrt{T H^{3}}\left(\log \frac{H}{\alpha}+d \kappa_{\lambda_{\min }}\right)+\sqrt{T H d \kappa_{\lambda_{\min }}}\left(\sqrt{d \lambda_{\max }}+\nu\right)\right)
$$

where $\kappa_{\lambda_{\min }}:=\log \left(1+K H^{2} /\left(\lambda_{\min }\right)\right)$ and $\lambda_{\min }=\min \left\{\lambda_{\min , p}, \lambda_{\min , r}\right\}, \lambda_{\max }=\max \left\{\lambda_{\max , p}, \lambda_{\max , r}\right\}$ and $\nu=\max \left\{\nu_{p}, \nu_{r}\right\}$.

Putting everything together, we have

$$
\mathcal{R}(T)=O\left(\sqrt{T H^{3}}\left(\log \frac{H}{\alpha}+d \kappa_{\lambda_{\min }}\right)+\sqrt{T H^{3} \log |\mathcal{A}|}+\sqrt{T H d \kappa_{\lambda_{\min }}}\left(\sqrt{d \lambda_{\max }}+\nu\right)\right)
$$

\section{Conclusion}

In this work, we took first step towards private RL algorithms with linear function approximations. We proposed both value-based and policy-based optimistic private RL algorithms and show that they enjoy sublinear regret in total number of steps while guaranteeing joint differential privacy (JDP). Moreover, these regret bounds are independent of the number of states and at most depends logarithmically on the number of actions in the policy optimization setting. Our established results build on a flexible procedure that also allows us to design general private RL algorithms with different privacy schemes under JDP and even under LDP guarantees by injecting Gaussian noise directly to each user's data. As discussed before, our work also opens the door to a series of interesting future works.

\section{Acknowledgement}

The author would like to thank Sayak Ray Chowdhury for insightful discussions and Quanquan Gu for the discussion on the concurrent work.

\section{References}

Lihong Li, Wei Chu, John Langford, and Robert E Schapire. A contextual-bandit approach to personalized news article recommendation. In Proceedings of the 19th international conference on World wide web, pages 661-670, 2010.

Yufan Zhao, Michael R Kosorok, and Donglin Zeng. Reinforcement learning design for cancer clinical trials. Statistics in medicine, 28(26):3294-3315, 2009. 
Akanksha Rai Sharma and Pranav Kaushik. Literature survey of statistical, deep and reinforcement learning in natural language processing. In 2017 International Conference on Computing, Communication and Automation (ICCCA), pages 350-354. IEEE, 2017.

Goren Gordon, Samuel Spaulding, Jacqueline Kory Westlund, Jin Joo Lee, Luke Plummer, Marayna Martinez, Madhurima Das, and Cynthia Breazeal. Affective personalization of a social robot tutor for children's second language skills. In Proceedings of the AAAI conference on artificial intelligence, volume 30, 2016.

Cynthia Dwork. Differential privacy: A survey of results. In International conference on theory and applications of models of computation, pages 1-19. Springer, 2008.

Aristide Tossou and Christos Dimitrakakis. Algorithms for differentially private multi-armed bandits. In Proceedings of the AAAI Conference on Artificial Intelligence, volume 30, 2016.

Aristide Tossou and Christos Dimitrakakis. Achieving privacy in the adversarial multi-armed bandit. In Proceedings of the AAAI Conference on Artificial Intelligence, volume 31, 2017.

Debabrota Basu, Christos Dimitrakakis, and Aristide Tossou. Differential privacy for multi-armed bandits: What is it and what is its cost? arXiv preprint arXiv:1905.12298, 2019.

Nikita Mishra and Abhradeep Thakurta. (nearly) optimal differentially private stochastic multi-arm bandits. In Proceedings of the Thirty-First Conference on Uncertainty in Artificial Intelligence, pages 592-601, 2015.

Xingyu Zhou and Jian Tan. Local differential privacy for bayesian optimization. arXiv preprint arXiv:2010.06709, 2020.

Roshan Shariff and Or Sheffet. Differentially private contextual linear bandits. arXiv preprint arXiv:1810.00068, 2018.

Giuseppe Vietri, Borja Balle, Akshay Krishnamurthy, and Steven Wu. Private reinforcement learning with pac and regret guarantees. In International Conference on Machine Learning, pages 9754-9764. PMLR, 2020 .

Evrard Garcelon, Vianney Perchet, Ciara Pike-Burke, and Matteo Pirotta. Local differentially private regret minimization in reinforcement learning. arXiv preprint arXiv:2010.07778, 2020.

David Silver, Julian Schrittwieser, Karen Simonyan, Ioannis Antonoglou, Aja Huang, Arthur Guez, Thomas Hubert, Lucas Baker, Matthew Lai, Adrian Bolton, et al. Mastering the game of go without human knowledge. nature, 550(7676):354-359, 2017.

Yan Duan, Xi Chen, Rein Houthooft, John Schulman, and Pieter Abbeel. Benchmarking deep reinforcement learning for continuous control. In International conference on machine learning, pages 1329-1338. PMLR, 2016.

William Yang Wang, Jiwei Li, and Xiaodong He. Deep reinforcement learning for nlp. In Proceedings of the 56th Annual Meeting of the Association for Computational Linguistics: Tutorial Abstracts, pages 19-21, 2018. 
Ronald J Williams. Simple statistical gradient-following algorithms for connectionist reinforcement learning. Machine learning, 8(3-4):229-256, 1992.

Sham M Kakade. A natural policy gradient. Advances in neural information processing systems, 14, 2001.

John Schulman, Sergey Levine, Pieter Abbeel, Michael Jordan, and Philipp Moritz. Trust region policy optimization. In International conference on machine learning, pages 1889-1897. PMLR, 2015.

John Schulman, Filip Wolski, Prafulla Dhariwal, Alec Radford, and Oleg Klimov. Proximal policy optimization algorithms. arXiv preprint arXiv:1707.06347, 2017.

Vijay R Konda and John N Tsitsiklis. Actor-critic algorithms. In Advances in neural information processing systems, pages 1008-1014. Citeseer, 2000.

Boyi Liu, Qi Cai, Zhuoran Yang, and Zhaoran Wang. Neural proximal/trust region policy optimization attains globally optimal policy. arXiv preprint arXiv:1906.10306, 2019.

Lingxiao Wang, Qi Cai, Zhuoran Yang, and Zhaoran Wang. Neural policy gradient methods: Global optimality and rates of convergence. arXiv preprint arXiv:1909.01150, 2019.

Qi Cai, Zhuoran Yang, Chi Jin, and Zhaoran Wang. Provably efficient exploration in policy optimization. In International Conference on Machine Learning, pages 1283-1294. PMLR, 2020.

Yonathan Efroni, Lior Shani, Aviv Rosenberg, and Shie Mannor. Optimistic policy optimization with bandit feedback. arXiv preprint arXiv:2002.08243, 2020.

Sayak Ray Chowdhury and Xingyu Zhou. Differentially private regret minimization in episodic markov decision processes. arXiv preprint arXiv:2112.10599, 2021.

Abhradeep Guha Thakurta and Adam Smith. (nearly) optimal algorithms for private online learning in full-information and bandit settings. Advances in Neural Information Processing Systems, 26:2733-2741, 2013.

Naman Agarwal and Karan Singh. The price of differential privacy for online learning. In International Conference on Machine Learning, pages 32-40. PMLR, 2017.

Bingshan Hu, Zhiming Huang, and Nishant A Mehta. Optimal algorithms for private online learning in a stochastic environment. arXiv preprint arXiv:2102.07929, 2021.

Touqir Sajed and Or Sheffet. An optimal private stochastic-mab algorithm based on optimal private stopping rule. In International Conference on Machine Learning, pages 5579-5588. PMLR, 2019.

John C Duchi, Michael I Jordan, and Martin J Wainwright. Local privacy and statistical minimax rates. In 2013 IEEE 54th Annual Symposium on Foundations of Computer Science, pages 429-438. IEEE, 2013.

Wenbo Ren, Xingyu Zhou, Jia Liu, and Ness B Shroff. Multi-armed bandits with local differential privacy. arXiv preprint arXiv:2007.03121, 2020.

Kai Zheng, Tianle Cai, Weiran Huang, Zhenguo Li, and Liwei Wang. Locally differentially private (contextual) bandits learning. arXiv preprint arXiv:2006.00701, 2020. 
Abhimanyu Dubey. No-regret algorithms for private gaussian process bandit optimization. In International Conference on Artificial Intelligence and Statistics, pages 2062-2070. PMLR, 2021.

Borja Balle, Maziar Gomrokchi, and Doina Precup. Differentially private policy evaluation. In International Conference on Machine Learning, pages 2130-2138. PMLR, 2016.

Baoxiang Wang and Nidhi Hegde. Privacy-preserving q-learning with functional noise in continuous state spaces. arXiv preprint arXiv:1901.10634, 2019.

Hajime Ono and Tsubasa Takahashi. Locally private distributed reinforcement learning. arXiv preprint arXiv:2001.11718, 2020.

Dongruo Zhou, Quanquan Gu, and Csaba Szepesvari. Nearly minimax optimal reinforcement learning for linear mixture markov decision processes. In Conference on Learning Theory, pages 4532-4576. PMLR, 2021a.

Jiafan He, Dongruo Zhou, and Quanquan Gu. Nearly optimal regret for learning adversarial mdps with linear function approximation. arXiv preprint arXiv:2102.08940, $2021 \mathrm{a}$.

Chonghua Liao, Jiafan He, and Quanquan Gu. Locally differentially private reinforcement learning for linear mixture markov decision processes. arXiv preprint arXiv:2110.10133, 2021.

Paul Luyo, Evrard Garcelon, Alessandro Lazaric, and Matteo Pirotta. Differentially private exploration in reinforcement learning with linear representation. arXiv preprint arXiv:2112.01585, 2021.

Alex Ayoub, Zeyu Jia, Csaba Szepesvari, Mengdi Wang, and Lin Yang. Model-based reinforcement learning with value-targeted regression. In International Conference on Machine Learning, pages 463-474. PMLR, 2020.

Dongruo Zhou, Jiafan He, and Quanquan Gu. Provably efficient reinforcement learning for discounted mdps with feature mapping. In International Conference on Machine Learning, pages 12793-12802. PMLR, 2021b.

Zeyu Jia, Lin Yang, Csaba Szepesvari, and Mengdi Wang. Model-based reinforcement learning with valuetargeted regression. In Learning for Dynamics and Control, pages 666-686. PMLR, 2020.

Jiafan He, Dongruo Zhou, and Quanquan Gu. Logarithmic regret for reinforcement learning with linear function approximation. In International Conference on Machine Learning, pages 4171-4180. PMLR, $2021 b$.

Dongsheng Ding, Xiaohan Wei, Zhuoran Yang, Zhaoran Wang, and Mihailo Jovanovic. Provably efficient safe exploration via primal-dual policy optimization. In International Conference on Artificial Intelligence and Statistics, pages 3304-3312. PMLR, 2021.

Chi Jin, Zhuoran Yang, Zhaoran Wang, and Michael I Jordan. Provably efficient reinforcement learning with linear function approximation. In Conference on Learning Theory, pages 2137-2143, 2020.

Lin Yang and Mengdi Wang. Sample-optimal parametric q-learning using linearly additive features. In International Conference on Machine Learning, pages 6995-7004. PMLR, 2019.

Cynthia Dwork, Aaron Roth, et al. The algorithmic foundations of differential privacy. 2014. 
Michael Kearns, Mallesh Pai, Aaron Roth, and Jonathan Ullman. Mechanism design in large games: Incentives and privacy. In Proceedings of the 5th conference on Innovations in theoretical computer science, pages 403-410, 2014.

Justin Hsu, Zhiyi Huang, Aaron Roth, Tim Roughgarden, and Zhiwei Steven Wu. Private matchings and allocations. SIAM Journal on Computing, 45(6):1953-1984, 2016.

Sayak Ray Chowdhury, Xingyu Zhou, and Ness Shroff. Adaptive control of differentially private linear quadratic systems. In 2021 IEEE International Symposium on Information Theory (ISIT), pages 485-490. IEEE, 2021.

Yasin Abbasi-Yadkori, Dávid Pál, and Csaba Szepesvári. Improved algorithms for linear stochastic bandits. Advances in neural information processing systems, 24:2312-2320, 2011.

Justin A Boyan. Least-squares temporal difference learning. In ICML, pages 49-56, 1999.

Steven J Bradtke and Andrew G Barto. Linear least-squares algorithms for temporal difference learning. Machine learning, 22(1):33-57, 1996.

T-H Hubert Chan, Elaine Shi, and Dawn Song. Private and continual release of statistics. ACM Transactions on Information and System Security (TISSEC), 14(3):1-24, 2011.

Mark Bun and Thomas Steinke. Concentrated differential privacy: Simplifications, extensions, and lower bounds. In Theory of Cryptography Conference, pages 635-658. Springer, 2016.

Tor Lattimore, Csaba Szepesvari, and Gellert Weisz. Learning with good feature representations in bandits and in rl with a generative model. In International Conference on Machine Learning, pages 5662-5670. PMLR, 2020.

Zhuoran Yang, Chi Jin, Zhaoran Wang, Mengdi Wang, and Michael I Jordan. On function approximation in reinforcement learning: Optimism in the face of large state spaces. arXiv preprint arXiv:2011.04622, 2020 .

Mojmír Mutnỳ and Andreas Krause. Efficient high dimensional bayesian optimization with additivity and quadrature fourier features. Advances in Neural Information Processing Systems 31, pages 9005-9016, 2019.

Daniele Calandriello, Luigi Carratino, Alessandro Lazaric, Michal Valko, and Lorenzo Rosasco. Gaussian process optimization with adaptive sketching: Scalable and no regret. In Conference on Learning Theory, pages 533-557. PMLR, 2019.

Albert Cheu, Adam Smith, Jonathan Ullman, David Zeber, and Maxim Zhilyaev. Distributed differential privacy via shuffling. In Annual International Conference on the Theory and Applications of Cryptographic Techniques, pages 375-403. Springer, 2019.

Roman Vershynin. Introduction to the non-asymptotic analysis of random matrices. arXiv preprint arXiv:1011.3027, 2010.

Michel Ledoux. The concentration of measure phenomenon. Number 89. American Mathematical Soc., 2001.

Beatrice Laurent and Pascal Massart. Adaptive estimation of a quadratic functional by model selection. Annals of Statistics, pages 1302-1338, 2000. 


\section{A Proof of Lemma 3.1}

Proof. Fix $h \in[H]$. Define $X_{h, p}^{k} \in \mathbb{R}^{(k-1) \times d_{1}}$ to be a matrix whose $j$-th row is $\left(\phi_{h}^{j}\left(s_{h}^{j}, a_{h}^{j}\right)\right)^{\top}$, and a 'noise' column vector $\eta_{h, p}^{k}$ whose $j$-th row is $V_{h+1}^{j}\left(s_{h+1}^{j}\right)-\left\langle\phi_{h}^{j}\left(s_{h}^{j}, a_{h}^{j}\right), \theta_{h, p}\right\rangle$. Then, we have for any $k \geq 1$,

$$
\begin{aligned}
& \theta_{h, p}-\widehat{\theta}_{h, p}^{k} \\
= & \theta_{h, p}-\left[\Lambda_{h, p}^{k}\right]^{-1} u_{h, p}^{k} \\
= & \theta_{h, p}-\left[\Lambda_{h, p}^{k}\right]^{-1}\left(\left(X_{h, p}^{k}\right)^{\top} X_{h, p}^{k} \theta_{h, p}+\left(X_{h, p}^{k}\right)^{\top} \eta_{h, p}^{k}+z_{h, p}^{k}\right) \\
= & \theta_{h, p}-\left[\Lambda_{h, p}^{k}\right]^{-1}\left(\Lambda_{h, p}^{k} \theta_{h, p}-Z_{h, p}^{k} \theta_{h, p}+\left(X_{h, p}^{k}\right)^{\top} \eta_{h, p}^{k}+z_{h, p}^{k}\right) \\
= & {\left[\Lambda_{h, p}^{k}\right]^{-1}\left(Z_{h, p}^{k} \theta_{h, p}-\left(X_{h, p}^{k}\right)^{\top} \eta_{h, p}^{k}-z_{h, p}^{k}\right) . }
\end{aligned}
$$

Therefore, we have

$$
\begin{aligned}
\left\|\theta_{h, p}-\widehat{\theta}_{h, p}^{k}\right\|_{\Lambda_{h, p}^{k}} & =\left\|Z_{h, p}^{k} \theta_{h, p}-\left(X_{h, p}^{k}\right)^{\top} \eta_{h, p}^{k}-z_{h, p}^{k}\right\|_{\left[\Lambda_{h, p}^{k}\right]^{-1}} \\
& \stackrel{(a)}{\leq}\left\|\left(X_{h, p}^{k}\right)^{\top} \eta_{h, p}^{k}\right\|_{\left[\Lambda_{h, p}^{k}\right]^{-1}}+\left\|Z_{h, p}^{k} \theta_{h, p}\right\|_{\left[\Lambda_{h, p}^{k}\right]^{-1}}+\left\|z_{h, p}^{k}\right\|_{\left[\Lambda_{h, p}^{k}\right]^{-1}} \\
& \stackrel{(b)}{\leq}\left\|\left(X_{h, p}^{k}\right)^{\top} \eta_{h, p}^{k}\right\|_{\left[\Lambda_{h, p}^{k}\right]^{-1}}+\left\|Z_{h, p}^{k} \theta_{h, p}\right\|_{\left[Z_{h, p}^{k}\right]^{-1}}+\left\|z_{h, p}^{k}\right\|_{\left[Z_{h, p}^{k}\right]^{-1}} \\
& =\underbrace{\left\|\left(X_{h, p}^{k}\right)^{\top} \eta_{h, p}^{k}\right\|_{\left[\Lambda_{h, p}^{k}\right]^{-1}}}_{\mathcal{B}_{1, p}}+\underbrace{\left\|\theta_{h, p}\right\|_{Z_{h, p}^{k}}+\left\|z_{h, p}^{k}\right\|_{\left[Z_{h, p}^{k}\right]^{-1}}}_{\mathcal{B}_{2, p}},
\end{aligned}
$$

where (a) follows from triangle inequality; (b) holds by $\Lambda_{h, p}^{k} \succeq Z_{h, p}^{k}$.

By the same argument, we have

$$
\left\|\theta_{h, r}-\widehat{\theta}_{h, r}^{k}\right\|_{\Lambda_{h, r}^{k}} \leq \underbrace{\left\|\left(X_{h, r}^{k}\right)^{\top} \eta_{h, r}^{k}\right\|_{\left[\Lambda_{h, r}^{k}\right]^{-1}}}_{\mathcal{B}_{1, r}}+\underbrace{\left\|\theta_{h, r}\right\|_{Z_{h, r}^{k}}+\left\|z_{h, r}^{k}\right\|_{\left[Z_{h, r}^{k}\right]^{-1}}}_{\mathcal{B}_{2, r}},
$$

where $X_{h, r}^{k} \in \mathbb{R}^{(k-1) \times d_{2}}$ is a matrix whose rows are $\left(\varphi\left(s_{h}^{1}, a_{h}^{1}\right)\right)^{\top}, \ldots,\left(\varphi\left(s_{h}^{k-1}, a_{h}^{k-1}\right)\right)^{\top}$ and $\eta_{h, r}^{k}$ is a 'noise' column vector whose $j$-th row is $r_{h}^{j}\left(s_{h}^{j}, a_{h}^{j}\right)-r_{h}\left(s_{h}^{j}, a_{h}^{j}\right)$.

Now, by Assumption 2, we have for any $\alpha \in(0,1]$, with probability at least $1-\alpha$, for all $k \in[K]$ and all $h \in[H]$

$$
\begin{aligned}
& \mathcal{B}_{1, p} \leq\left\|\left(X_{h, p}^{k}\right)^{\top} \eta_{h, p}^{k}\right\|_{\left[G_{h, p}^{k}+\lambda_{\min , p}\right]^{-1}} \quad \text { and } \quad \mathcal{B}_{1, r} \leq\left\|\left(X_{h, r}^{k}\right)^{\top} \eta_{h, r}^{k}\right\|_{\left[G_{h, r}^{k}+\lambda_{\min , r}\right]^{-1}} \\
& \mathcal{B}_{2, p} \leq \sqrt{d_{1} \lambda_{\max , p}}+\nu_{p} \quad \text { and } \quad \mathcal{B}_{2, r} \leq \sqrt{d_{2} \lambda_{\max , r}}+\nu_{r},
\end{aligned}
$$

where $G_{h, p}^{k}:=\left(X_{h, p}^{k}\right)^{\top}\left(X_{h, p}^{k}\right)$ and $G_{h, r}^{k}:=\left(X_{h, r}^{k}\right)^{\top}\left(X_{h, r}^{k}\right)$. Moreover, we also used the assumption in Definition 1 that $\left\|\theta_{h, p}\right\| \leq \sqrt{d_{1}}$ and $\left\|\theta_{h, r}\right\| \leq \sqrt{d_{2}}$.

We are only left with two noise terms, which can be bounded by the standard self-normalized inequality (cf. (Abbasi-Yadkori et al., 2011, Theorem 1)). In particular, first note that $j$-th element of the 'noise' vector 
$\eta_{h, p}^{j}$ is $V_{h+1}^{j}\left(s_{h+1}^{j}\right)-\left\langle\phi_{h}^{j}\left(s_{h}^{j}, a_{h}^{j}\right), \theta_{h, p}\right\rangle=V_{h+1}^{j}\left(s_{h+1}^{j}\right)-\mathbb{E}_{s^{\prime} \sim \mathbb{P}\left(\cdot \mid s_{h}^{j}, a_{h}^{j}\right)} V_{h+1}^{j}\left(s^{\prime}\right)$, which holds by the linear kernel MDP model in Assumption 1. Note that, conditioning on $\mathcal{F}_{j, h, 1}, \eta_{h, p}^{j}$ is a zero-mean random variable. This holds by the fact that conditioning on $\mathcal{F}_{j, h, 1}$, the only randomness comes from $s_{h+1}^{j}$. Moreover, since $V_{h+1}^{j} \in[0, H]$, conditioning on $\mathcal{F}_{j, h, 1}, \eta_{h, p}^{j}$ is an $H / 2$-sub-Gaussian random variable. Also, $\eta_{h, p}^{j}$ is $\mathcal{F}_{j, h, 2}$ measurable and $\phi_{h}^{j}\left(s_{h}^{j}, a_{h}^{j}\right)$ is $\mathcal{F}_{j, h, 1}$ measurable by Definition 7.1. Similarly, we can see that the noise $\eta_{h, r}^{j}$ is a $1 / 2$-sub-Gaussian random variable conditioning on $\mathcal{F}_{j, h-1,2}$ as $\pi^{j}$ is measurable with respect to $\mathcal{F}_{j, h, 1}$ and hence conditioning on $\mathcal{F}_{j, h-1,2}$, the only randomness comes from the reward realization $r_{h}^{j}\left(s_{h}^{j}, a_{h}^{j}\right) \in$ $[0,1]$. Moreover, $\eta_{h, r}^{j}$ is $\mathcal{F}_{j, h, 1}$ measurable and $\varphi\left(s_{h}^{j}, a_{h}^{j}\right)$ is $\mathcal{F}_{j, h-1,2}$ measurable. With such choices of filtrations, one can now apply standard self-normalized inequality to obtain that for any fixed $\alpha \in(0,1]$, with probability at least $1-3 \alpha$, for all $k \in[K]$ and $h \in[H]$

$$
\mathcal{B}_{1, p} \leq \frac{H}{2} \sqrt{2 \log \left(\frac{H}{\alpha}\right)+\log \frac{\operatorname{det}\left(G_{h, p}^{k}+\lambda_{\min , p} I\right)}{\operatorname{det}\left(\lambda_{\min , p} I\right)}}
$$

and

$$
\mathcal{B}_{1, r} \leq \frac{1}{2} \sqrt{2 \log \left(\frac{H}{\alpha}\right)+\log \frac{\operatorname{det}\left(G_{h, r}^{k}+\lambda_{\min , r} I\right)}{\operatorname{det}\left(\lambda_{\min , r} I\right)}} .
$$

Then, we can further bound the two terms by using the trace-determinant lemma (cf. (Abbasi-Yadkori et al., 2011, Lemma 11)) and the boundedness assumptions in Assumption 1, that is, for all all $h \in[H]$ and $k \in[K],\left\|\phi_{h}^{k}\left(s_{h}^{k}, a_{h}^{k}\right)\right\| \leq \sqrt{d_{1}} H$ and $\left\|\varphi\left(s_{h}^{k}, a_{h}^{k}\right)\right\| \leq 1$. In particular, we obtain that with probability at least $1-3 \alpha$, for all $k \in[K]$ and $h \in[H]$

$$
\mathcal{B}_{1, p} \leq \frac{H}{2} \sqrt{2 \log \left(\frac{H}{\alpha}\right)+d_{1} \log \left(1+\frac{K H^{2}}{\lambda_{\min , p}}\right)}
$$

and

$$
\mathcal{B}_{1, r} \leq \frac{1}{2} \sqrt{2 \log \left(\frac{H}{\alpha}\right)+d_{2} \log \left(1+\frac{K}{d_{2} \lambda_{\min , r}}\right)} .
$$

Finally, putting everything together, we have for any fixed $\alpha \in(0,1]$, with probability at least $1-3 \alpha$ for all $k \in[K]$ and $h \in[H]$,

$$
\left\|\theta_{h, p}-\widehat{\theta}_{h, p}^{k}\right\|_{\Lambda_{h, p}^{k}} \leq \frac{H}{2} \sqrt{2 \log \left(\frac{H}{\alpha}\right)+d_{1} \log \left(1+\frac{K H^{2}}{\lambda_{\min , p}}\right)}+\sqrt{d_{1} \lambda_{\max , p}}+\nu_{p}
$$

and

$$
\left\|\theta_{h, r}-\widehat{\theta}_{h, r}^{k}\right\|_{\Lambda_{h, r}^{k}} \leq \frac{1}{2} \sqrt{2 \log \left(\frac{H}{\alpha}\right)+d_{2} \log \left(1+\frac{K}{d_{2} \lambda_{\min , r}}\right)}+\sqrt{d_{2} \lambda_{\max , r}}+\nu_{r}
$$




\section{B Useful Lemmas}

The following lemmas are the key components to prove Theorem 6.5.

Lemma B.1 (Slepian's inequality; Lemma 5.33 in Vershynin (2010)). Consider two Gaussian processes $\left(X_{t}\right)_{t \in T}$ and $\left(Y_{t}\right)_{t \in T}$ whose increments satisfy the inequality $\mathbb{E}\left[\left|X_{s}-X_{t}\right|^{2}\right] \leq \mathbb{E}\left[\left|Y_{s}-Y_{t}\right|^{2}\right]$ for all $s, t \in$ $T$, then, $\mathbb{E}\left[\sup _{t \in T} X_{t}\right] \leq \mathbb{E}\left[\sup _{t \in T} Y_{t}\right]$.

Lemma B.2 (Concentration in the Gauss space). Let $f$ be a real valued 1-Lipschitz function on $\mathbb{R}^{n}$. Let $X$ be a Gaussian random vector in $\mathbb{R}^{n}$ whose entries $X_{i}$ is i.i.d $\mathcal{N}\left(0, \sigma^{2}\right)$. Then, for every $t \geq 0$,

$$
\mathbb{P}\{f(X)-\mathbb{E}[f(X)] \geq t\} \leq \exp \left(-\frac{t^{2}}{2 \sigma^{2}}\right)
$$

Proof. This result directly follows from Proposition 2.18 in Ledoux (2001) by considering $\gamma$ as the Gaussian measure on $\mathbb{R}^{n}$ with density of a multivariate normal distribution, i.e., $\mathcal{N}\left(0, \sigma^{2} I\right)$ and hence $c$ in Proposition 2.18 of Ledoux (2001) is equal to $\frac{1}{\sigma^{2}}$.

Lemma B.3 (Operator norm of Gaussian random matrices). Let $A$ be an $m \times n$ random matrix whose entries $A_{i j}$ are i.i.d Gaussian random variables $\mathcal{N}\left(0, \sigma^{2}\right)$. Then, for any $t>0$, we have

$$
\mathbb{P}\{\|A\| \geq \sigma(\sqrt{n}+\sqrt{m})+t\} \leq \exp \left(-\frac{t^{2}}{2 \sigma^{2}}\right) .
$$

Proof. We will first show that

$$
\mathbb{E}[\|A\|] \leq \sigma(\sqrt{m}+\sqrt{n})
$$

by using Lemma B.1. To this end, note that the operator norm of $A$ can be computed as follows.

$$
\|A\|=\max _{u \in S^{n-1}, v \in S^{m-1}}\langle A u, v\rangle .
$$

where $S^{n-1}$ and $s^{m-1}$ are unit spheres. Thus, $\|A\|$ can be regarded as the supremum of the Gaussian process $X_{u, v}:=\langle A u, v\rangle$ indexed by the pair of vectors $(u, v) \in S^{n-1} \times S^{m-1}$. Let us define $Y_{u, v}:=\langle g, u\rangle+\langle h, v\rangle$ where $g \in \mathbb{R}^{n}$ and $h \in \mathbb{R}^{m}$ are independent Gaussian random vectors whose entries are i.i.d Gaussian random variables $\mathcal{N}\left(0, \sigma^{2}\right)$. Now, we compare the increments of these two Gaussian processes for every $(u, v),\left(u^{\prime}, v^{\prime}\right) \in S^{n-1} \times S^{m-1}$.

$$
\begin{aligned}
\mathbb{E} & {\left[\left|X_{u, v}-X_{u^{\prime}, v^{\prime}}\right|^{2}\right] } \\
= & \sigma^{2} \sum_{i=1}^{n} \sum_{j=1}^{m}\left|u_{i} v_{j}-u_{i}^{\prime} v_{j}^{\prime}\right|^{2} \\
= & \sigma^{2}\left\|u v^{T}-u^{\prime} v^{T}\right\|_{F}^{2} \\
= & \sigma^{2}\left\|\left(u-u^{\prime}\right) v^{T}+u^{\prime}\left(v-v^{\prime}\right)^{T}\right\|_{F}^{2} \\
= & \sigma^{2}\left(\left\|u-u^{\prime}\right\|_{2}^{2}+\left\|v-v^{\prime}\right\|_{2}^{2}\right) \\
& +2 \sigma^{2} \operatorname{trace}\left(v\left(u-u^{\prime}\right)^{T} u^{\prime}\left(v-v^{\prime}\right)^{T}\right) \\
= & \sigma^{2}\left(\left\|u-u^{\prime}\right\|_{2}^{2}+\left\|v-v^{\prime}\right\|_{2}^{2}+2\left(u^{T} u^{\prime}-1\right)\left(1-v^{T} v^{\prime}\right)\right) \\
\leq & \sigma^{2}\left(\left\|u-u^{\prime}\right\|_{2}^{2}+\left\|v-v^{\prime}\right\|_{2}^{2}\right) \\
= & \mathbb{E}\left[\left|Y_{u, v}-Y_{u^{\prime}, v^{\prime}}\right|^{2}\right] .
\end{aligned}
$$


Therefore, Lemma B.1 applies here, and hence

$$
\begin{aligned}
\mathbb{E}[\|A\|] & =\mathbb{E}\left[\max _{(u, v)} X_{u, v}\right] \leq \mathbb{E}\left[\max _{(u, v)} Y_{u, v}\right] \\
& =\mathbb{E}\left[\|g\|_{2}\right]+\mathbb{E}\left[\|h\|_{2}\right] \leq \sigma(\sqrt{m}+\sqrt{n}) .
\end{aligned}
$$

Finally, note that $\|A\|$ is a 1-Lipschitz function of $A$ when considered as a vector in $\mathbb{R}^{m n}$. Then, consider the function $f$ in Lemma B.2 as the operator norm, we directly obtain the result.

Lemma B.4 (Operator norm of symmetric Gaussian random matrices). Let A be an $n \times n$ random symmetric matrix whose entries $A_{i j}$ on and above the diagonal are i.i.d Gaussian random variables $\mathcal{N}\left(0, \sigma^{2}\right)$. Then, for any $t>0$, we have

$$
\|A\| \leq 4 \sigma \sqrt{n}+2 t
$$

with probability at least $1-2 \exp \left(-\frac{t^{2}}{2 \sigma^{2}}\right)$.

Proof. Decompose the matrix $A$ into the upper triangular matrix $A^{+}$and the lower triangular matrix $A^{-}$ such that $A=A^{+}+A^{-}$. Note that without loss of generality, the entries on the diagonal are in $A^{+}$. Then, apply Lemma B.3 to both $A^{+}$and $A^{-}$, and by a union bound, we have that the following inequalities hold simultaneously

$$
\left\|A^{+}\right\| \leq 2 \sigma \sqrt{n}+t \quad \text { and } \quad\left\|A^{-}\right\| \leq 2 \sigma \sqrt{n}+t
$$

with probability at least $1-2 \exp \left(-\frac{t^{2}}{2 \sigma^{2}}\right)$ for any $t>0$. Finally, by triangle inequality $\|A\| \leq\left\|A^{+}\right\|+\left\|A^{-}\right\|$, we prove the result.

Lemma B.5 (Concentration of chi-square; Corollary to Lemma 1 of Laurent and Massart (2000)). Let U be a $\chi^{2}$ statistic with $D$ degrees of freedom. Then, for any positive $x$,

$$
\mathbb{P}\{U \geq D+2 \sqrt{D x}+2 x\} \leq \exp (-x) .
$$

